\title{
6 Schule Hinter Gärten in Riehen - Kunstwerk und postfordistisches Produktionsgebäude
}

Das Schulhaus Hinter Gärten in der Gemeinde Riehen im Kanton Basel-Stadt stellt eine Schulanlage dar, die den aktuellen Diskurs einer zeitgemäßen, die pädagogischen Trends entsprechenden Schulhausarchitektur abbildet und manifestiert. Das Schulhaus wurde nicht als Erweiterungs- oder Umbau geplant wurde, sondern von Grund auf für eine wachsende bzw. neu entstehende Siedlung konzipiert.

\subsection{Gemeinde Riehen - Urbane Platzknappheit auf dem Lande}

Auf dem Luftbild (Abb. 12) ist ein Ausschnitt des Siedlungsraums Riehen zu sehen, der durch Wohnhäuser in einer ländlichen Gegend dominiert wird. Die Bahnlinie und die fast parallel verlaufende Straße zeigen, dass sich das Dorf an einer Verkehrsachse befindet, die in Nord-Süd-Richtung verläuft. Auffallend ist die Mischung aus landwirtschaftlicher Nutzung und Gebäuden, die mehrheitlich Mehrfamilienhäuser sind. Es sind jedoch auch Straßenzüge mit Einfamilienhäusern erkennbar. Sowohl die Mehr- als auch die Einfamilienhäuser sind großzügig gestaltet. Sie sind meist in umgebende Grünflächen eingebettet, außerdem sind etliche Schwimmbecken erkennbar.

In der Siedlung können unterschiedliche Bereiche identifiziert werden, die jeweils verschiedenen Bauetappen zuzuweisen sind: Unten links lässt die Straßenanordnung auf einen historisch-traditionell gewachsenen Dorfkern schließen, der selbst nicht mehr im Bild ist. Die Straße führt zu einem Gebiet oben links, welches als zusammenhängendes Gebiet geplant und bebaut wurde. Nordöstlich und südöstlich ziehen sich entlang kleinerer Straßenzüge eine Reihe von vorwiegend Ein- und wenigen Mehrfamilienhäusern, die sich durch großzügige Gartenanlagen auszeichnen.

Der größte und durch seine Farbe auffälligste Gebäudekomplex ist das zentral ins Bild gerückte zweiteilige Gebäude. Es ist an einem Acker platziert. Auf dieses Gebäude soll im Folgenden näher eingegangen werden: das Schulhaus 
Hinter Gärten. Zunächst wird jedoch seine Positionierung innerhalb der Siedlung thematisiert.

Im Siedlungsbild sind die älteren Gebäude und die Infrastrukturen wie Straßen und Bahntrasse entlang der Nord-Süd-Achse ausgerichtet. Die neueren Gebäude wurden entlang der Straßenzüge in einer Ost-West-Richtung erstellt. Die Siedlung entwickelt sich aktuell von West nach Ost, wobei sich in der Mitte des Bildes ein Streifen erhalten hat, der aus landwirtschaftlichen Flächen, Wald und Wiese besteht. Hier sticht insbesondere die Ackerfläche im Zentrum des Bildes ins Auge.

Die Fläche ist viel zu klein, um ein landwirtschaftliches Auskommen zu generieren. Zudem fehlen Gebäude, die zu einem Landwirtschaftsbetrieb passen. Es ist lediglich ein kleines scheunenartiges Gebäude ersichtlich. Außerdem ist das Gebiet von unterschiedlichen Gebäuden umrandet, die offensichtlich nicht landwirtschaftlich genutzt werden. Die Fläche erscheint als eine übrig gebliebene Scholle zwischen zwei zusammenwachsenden Siedlungsgebieten. Es ist ein Strukturwandel von einem landwirtschaftlich-agrarisch ausgerichteten Dorf zu einer vorstädtischen Agglomerationssiedlung festzustellen wobei der Acker das letzte Überbleibsel, ein Relikt zu sein scheint. Die Gebäude im Ausschnitt sind fast ausschließlich Wohngebäude. Es sind aneinandergereihte Häuser, die in ihrer Summe ein planloses Siedlungsbild ohne erkennbaren inneren Zusammenhalt ergeben: Weder sind öffentliche Flächen oder Plätze noch ein soziales oder ökonomisches Zentrum erkennbar.

Im Vergleich zu einer Stadt ist das Gebiet wenig dicht besiedelt. Die älteren Gebäude sind größere Mehrfamilienhäuser. Wahrscheinlich sind sie ab den 1960er Jahren entstanden. Dabei ist auch ein Unterschied in der Art der Häuser auszumachen. Die neueren Gebäude sind vorwiegend Ein- oder aneinandergebaute Zweifamilienhäuser. Es sind individuelle Wohnsitze mit privaten Gärten. Die Siedlung bietet - abgesehen vom Acker - nicht viel an erkennbarem ökonomischem Potenzial. Die Häuser, die als private Wohnsitze mit viel Erholungsund Reproduktionsräumen konzipiert sind, verweisen darauf, dass es vorwiegend wohlhabende mobile Pendler_innen sind, die hier angesiedelt sind. Das viele Grün, der hohe Baumbestand und die zahlreichen Swimmingpools unterstreichen dies. 


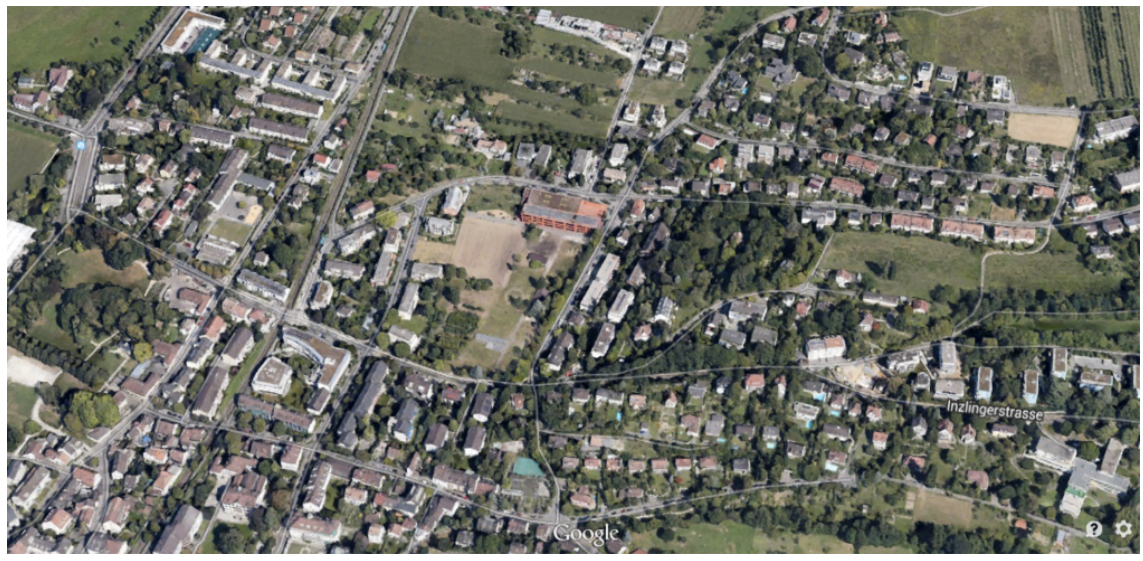

Abb. 12: Luftbild Gemeinde Riehen (BS). (C) Google Maps 2015

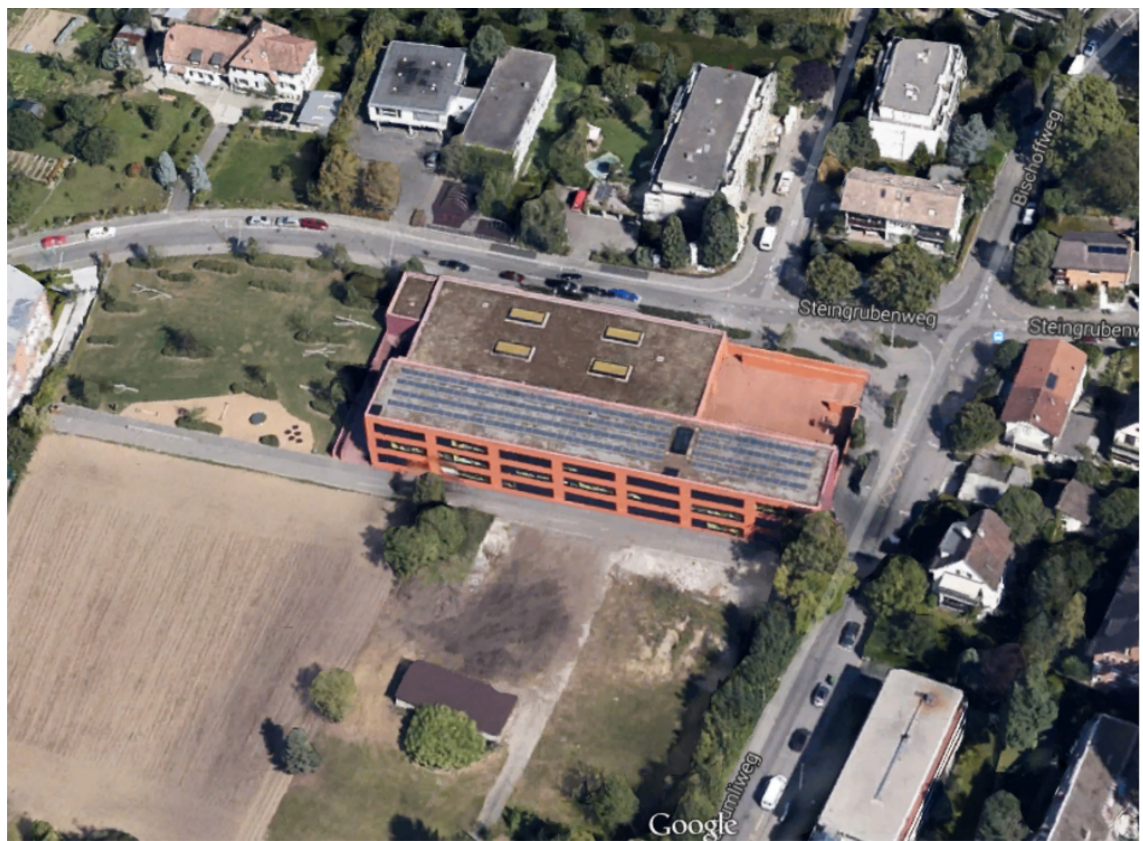

Abb. 13: Luftbild Schulhaus Hinter Gärten. Riehen (BS). (C) Google Maps 2015 
Der Titel nimmt auf, dass das Schulhaus an der Stelle zweier fremder Kulturen zu liegen kommt und diese verbindet. Es ist also zu fragen, ob und wenn ja wie eine Verbindung geschaffen wird. Denn als erweiterte Schulanlage hat das Gebäude den gegenteiligen Effekt. Die Schüler_innen der umgebenden Bebauung gehen nun nicht mehr mit Kindern aus anderen Vierteln in die zwei bereits bestehenden Schulhäuser, die im Dorfzentrum liegen. Sie sind nun sozialräumlich gesehen unter ihresgleichen in einem ,eigenen' Schulhaus. Damit vollzieht sich eine sozialräumliche Separierung: Die Schüler_innen sind nun auch in der Schule in einer Peer Group, die sich als relativ homogen erweist, wie sich aus der Siedlungsstruktur rekonstruieren lässt.

Das Schulhaus ist platzsparend in die äußerste Ecke des freien Geländes gesetzt. Das Schulhaus schafft damit wenig bis keinen einladenden, nutzbaren öffentlichen Raum. Dies ist bemerkenswert, da der Platz dafür vorhanden ist. Auch hat das Schulhaus im Vergleich zu den privaten Eigenheimen wenig Freifläche. Es geht weder eine Verbindung mit der Siedlung ein, noch kann es einen Bezugspunkt für die Siedlung setzen, was zugegebenermaßen in dieser losen Aneinanderreihung auch kein einfaches Unterfangen wäre. Das Schulhaus reiht sich ein in ein Siedlungsgefüge, welches wenig eigene (Siedlungs-)Identität erkennen lässt, sondern aus zufällig aneinandergereihten, zusammenhangslos verwirklichten ,individuellen ${ }^{41}$ Träumen von Wohneigentum besteht. Das Schulhaus reproduziert diesen Selbstbezug - oder eher den Rückzug ins private Heim - mehr, als es Gegensteuer gibt, indem ein öffentlicher Raum geschaffen wird. Dies ist insofern bemerkenswert, da die (einmalige) Chance vorhanden ist, eine großzügig bemessene Fläche für verschiedene Nutzungen der Öffentlichkeit oder auch der Schule zuzuführen, wie es etwa bei der Schule Schmitten (Kapitel 7) der Fall ist. Denn im Gegensatz zu vielen Schulhäusern in urbanen Gegenden ist hier der Platz vorhanden, um eine Schule zu realisieren, die umfassende Infrastrukturen im Hinblick auf Tagesstrukturen oder auch auf eine differenzierende Pädagogik ermöglichen könnte. Es gibt Raum für Gärten, Werkstätte, (Klein-) Tier-Haltung usw., was alles räumliche Settings darstellt, die normativ in den pädagogischen Diskursen für die ,gute Schule“ und als Anforderungen ,zukünf-

41 Das Luftbild zeigt, wie auch unter der Prämisse individuell verwirklichter Eigentumsträume die Behausungen sich schlussendlich ähnlich sind (vgl. auch Bourdieu 2011). 
tiger Schulen“ (Grimm 2006; Kahl 2005; Walden \& Borrelbach 2010) ins Feld geführt werden. Die Schule hätte als ein siedlungsbaulicher Bezugspunkt mit öffentlichem Park und Landreserven für zukünftige Anforderungen an Bildungsbauten (z. B. Tagesschulen) gebaut werden können, wie sie in den Konzepten und Strategien der kantonalen Bildungspolitik und der Bildungsdirektionen als zukünftige Anforderungen formuliert werden. Das Potenzial, das dem Gebiet innewohnt, wird nicht eingelöst. Im Gegenteil muss angenommen werden, dass der, durch die sparsame Platzierung der Schule, ,gewonnene' Raum voraussichtlich für die Verwirklichungen privater Räume genutzt werden wird. Im Folgenden wird das Schulhaus genauer betrachtet.

\subsection{Schulhaus Hinter Gärten - Architektur als krisenevozierendes Werk}

Der Wettbewerb für das Schulhaus Hinter Gärten wird 1999 ausgeschrieben. Vorgaben im Wettbewerb sind: ein der Altersstufe gerechtes leichtes Auffinden der Klassenräume; separate Zugänge zur Aula und den beiden Turnhallen sowie die Vorgabe, dass in den Klassenzimmern sowohl ein direkter Zugang zu Gruppenarbeitsräumen als auch die akustische Schließung dieser Räume gewährleistet sein müssen. Das Siegerprojekt wird unter dem Namen east meets west von den Architekten Marques AG eingereicht. 2006 wird das Schulhaus eingeweiht. Das für das Schulhaus dominante Farbkonzept wird vom Künstler Jörg Niederberger ausgearbeitet.

Kurze Zeit nach der Fertigstellung wurden bereits zusätzliche Klassenzimmer notwendig. In zwei provisorisch erstellten Barackenbauten kommen weitere acht Räume hinzu. Bei der Datenerhebung im Januar 2015 waren die Erweiterungsbaracken eben fertiggestellt. Auf den verwendeten Luftbildern sind sie nicht ersichtlich. Sie werden nicht mitanalysiert. Auf Teilen der verwendeten Fotografien sind sie aber am Rande erkennbar.

Ersichtlich ist ein Gebäudekomplex, der aus zwei großen und einem kleinen Volumen besteht (Abb. 14-19). 


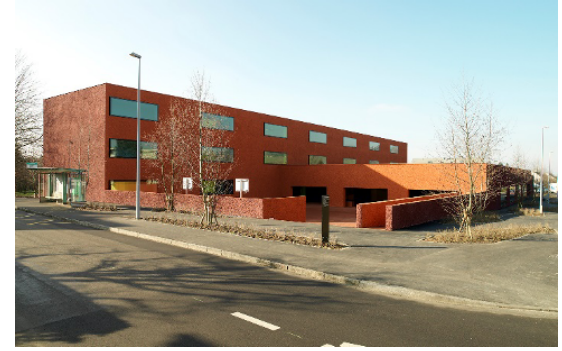

Abb. 14: Hinter Gärten. (C) Heinz Unger, Zürich

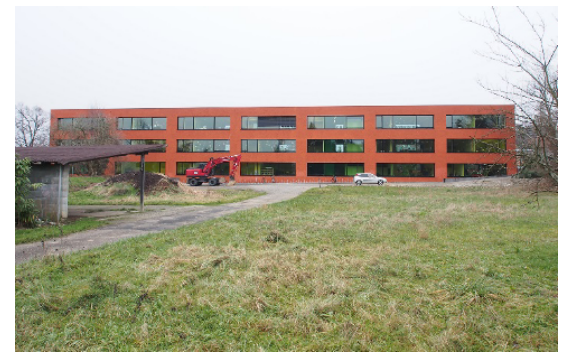

Abb. 16: Hinter Gärten. Ansicht Süd

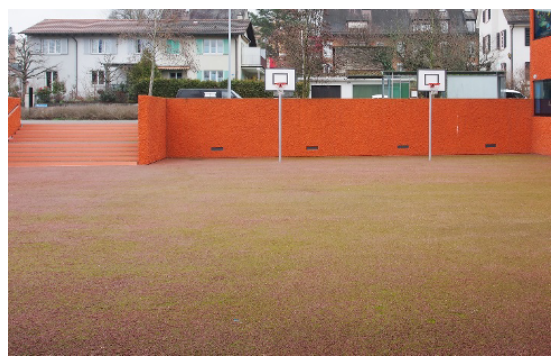

Abb. 18: Hinter Gärten. Pausenplatz

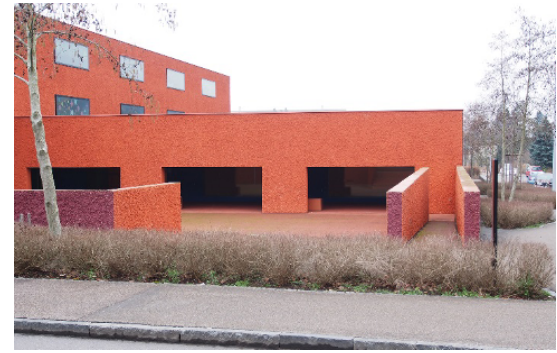

Abb. 15: Hinter Gärten. Eingangsbereich Nordost

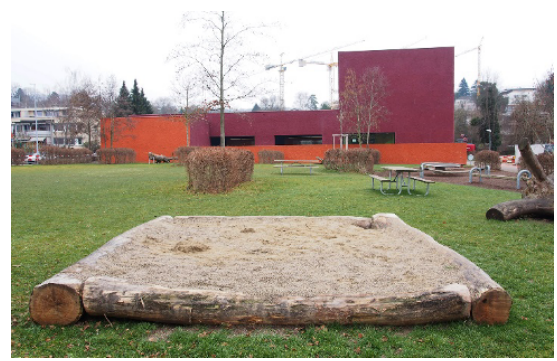

Abb. 17: Hinter Gärten. Pausenplatz

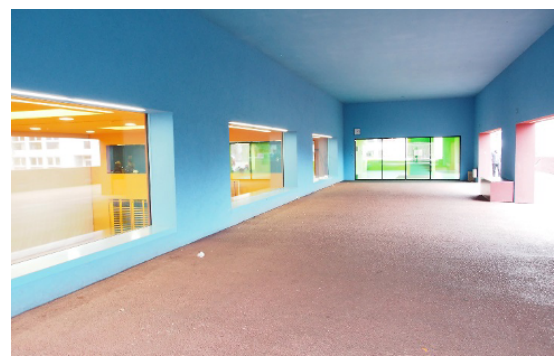

Abb. 19: Hinter Gärten. Eingangsbereich

Ein längliches dreigeschossiges Gebäudevolumen mit sieben Fensterachsen stellt einen langen Riegel dar. Davor ist ein eingeschossiges großes Gebäudevolumen mit Oberlichtern angebaut. In dem vorgelagerten Gebäude sind die Erschließungen angebracht. Im Nordwesten daran angehängt befindet sich ein dritter, sehr kleiner Baukörper. Im Nordosten kreieren die beiden Gebäude einen tiefer gelegenen Platz. Er ist in derselben Farbe wie das Gebäude gehalten. Auch im Wes- 
ten des Gebäudes zeigt sich eine Erschließung, dessen Bodenbelag dieselbe Farbe hat. Beide Erschließungen der Schulanlagen sind von einer auffälligen Doppelmauer flankiert. Diese umfasst eine Rampe und ist von den Treppen abgegrenzt. In den Detailaufnahmen wird ersichtlich, dass das Gebäude nicht nur eine auffällige Farbe besitzt. Das Gebäude ist zweifarbig, wobei die Fassaden jeweils einfarbig gestaltet sind und sich die Farben je nach Ausrichtung abwechseln. Beim lang gezogenen Bau sind die Stirnseiten in Magenta und die Breitseiten in Rostrot gehalten. Bei der angebauten Halle sind es die Nord- und die Westseite, die in Magenta gehalten sind. Die Ostseite ist rostrot. Zudem sind die Bodenflächen innerhalb der rechteckigen Grundfläche ebenfalls rostrot eingefärbt.

Planungsgrundlage war offensichtlich ein rot eingefärbtes Rechteck. Darauf wird mit den Gebäudevolumen eine Rhythmisierung zwischen Hoch und Tief, zwischen Vorne und Hinten, zwischen Breite und Tiefe erstellt. Die abwechselnde dichotome Rhythmisierung wird von den beiden Farbtönen aufgenommen und verstärkt. Gegen Norden ist dem langen, schmalen und hohen Trakt ein niedriges, breites und tiefes Gebäudevolumen vorgelagert.

Die Formen des gesamten Komplexes sind minimalistisch und schnörkellos gehalten. Neben der Farbgebung und den Gebäudekörpern sind es die Öffnungen, die den Bau gestalten. In den Gebäudevolumen sind auf jeder Seite gleichartige Öffnungen eingelassen. Im länglichen Trakt sind pro Stockwerk sieben Fenster gleichmäßig verteilt, was auf sieben gleichartige Räume verweist und dem Gebäude durch die ungerade Anzahl eine Mitte gibt. Nach Süden sind die Fenster etwas größer als im Norden, was einen hohen Lichteinfall garantiert. Die Räume sind nach Süden hin ausgerichtet. Hier ist auch exemplarisch zu sehen, wie hermetisch abgeschlossen das längliche Gebäudevolumen ist. Die Fenster können nicht geöffnet werden.

Das niedrige Gebäude hat Einlassungen auf drei Seiten. An der Ostseite und Westseite befinden drei breite Öffnungen, die eine - nicht gegebene - Durchlässigkeit suggerieren. Auf der Nordseite gegen die Straße ist die Erschließung des Gebäudes in Form einer breiten Öffnung. Neben dem Eingang sind vier Fenster. Die Erschließungen sind so angebracht, dass der hintere Längstrakt über das vordere Gebäudevolumen betreten wird.

Der Gebäudekomplex ist auf drei Seiten von Straßen umgeben, wobei die Straße auf der Südseite eine Sackgasse ist. Im Westen schließt sich an das Ge- 
bäude eine Außenfläche an. Verschiedene Elemente auf ihr nehmen die OstWest-Ausrichtung des Gebäudes auf: Hecken und liegende Baumstämme.

Der Gebäudekomplex zeichnet sich in erster Linie durch seine außeralltägliche Farbgebung aus. Damit löst das Gebäude eine Erfahrungskrise aus. Es drängt sich durch seine Farbigkeit zwingend in den Aufmerksamkeitsfokus des Betrachters. Dabei ist es nicht ,nur' die Farbe. Es ist eine Mischung des Farbtons, des monochromen Farbauftrags und des Sättigungsgrads, welche die Wirkung eines außeralltäglichen Gegenstands entfaltet.

Die Farbintensität und die Außeralltäglichkeit dieser Farbintensität von Gebäuden hat zur Folge, dass beim Schulhaus Hinter Gärten kaum vorstellbar ist, dass das Gebäude von zufällig vorbeikommenden Personen nicht wahrgenommen wird; dem Betrachter drängt es sich zwangsläufig auf. Die Farbgebung erheischt eine Aufmerksamkeit, die dem Gebäude aus seiner Form nicht in dieser Art und Weise zuteilwird. Das Gebäude stellt damit in der Diskrepanz einer außeralltäglichen Farbgebung und der Nüchternheit seiner Form eine Spannung her. Das Haus verlangt danach, seine Farbigkeit und seine Morphologie gesondert zu betrachten. Zuerst wird diskutiert, was das Gebäude durch seine Farbe (re)präsentiert. Danach wird die Positionalität und die damit einhergehende Innen-Außen-Abgrenzung genauer betrachtet sowie auf die eigentliche Architektur eingegangen.

Das Schulhaus drängt sich über die krisenhaft expressive Farbgestaltung in die Aufmerksamkeit: Es stellt eine Erfahrungskrise durch die Farbe dar. Um dies konturierter herauszuarbeiten, kann man sich überlegen, welche Gebäude durch eine intensive Farbgebung charakterisiert sind. Die Farbintensivität von Gebäuden ist entweder baustoff- oder kulturabhängig, oder die beiden Elemente bilden eine Einheit. Satte, leuchtende Farbanstriche sind etwa in Skandinavien nicht außergewöhnlich. Auch die sardische Stadt Bosa ist für ihre farbigen Häuser bekannt. Beiden Beispielen ist gemein, dass die Farbigkeit einzelner Gebäude nicht krisenevozierend ist, sondern routinehaft ein Bauensemble bestimmt.

Farbige Gebäude, die aus der Umgebung herausstechen, vermitteln durch Farbe eine Botschaft, zum Beispiel als Marketingstrategie. Firmen können ihren Namen mit einer Farbe verbinden und damit über eine spezifische Farbe ihre Präsenz vermitteln. Die Farbgebung von Gebäuden ist in der Schweiz streng reglementiert und bedarf einer Begutachtung und Bewilligung der Denkmalpfle- 
ge. Ein Gebäude darf nur dann neongelb sein, wenn es sich entweder in die Umgebung einpasst oder ihm ein künstlerischer Wert zugeschrieben wird.

Dies ist bei Gebäuden dann gehäuft der Fall, wenn es sich um Kulturbauten oder um Bildungsbauten handelt. Die Botschaft dabei ist, dass die Gebäude durch die Farbgebung - meist einhergehend mit irgendeiner Art herausragender Architektur - die Funktionalität des Gebäudes, z. B. eines Museums, repräsentiert. Damit nehmen die Gebäude per se den Charakter eines Kunstwerks für sich in Anspruch. Oft geht die Farbigkeit einer Architektur mit einer avantgardistischen Architektur einher, deren Bedeutung und Wirkung über den Radius der visuellen Sichtbarkeit hinaus verstärkt werden soll. Das Gebäude bzw. die bewohnende Institution soll über ihre Architektur eine ikonische Bekanntheit durch Publikationen und Ähnliches erlangen.

Diese Lesart reproduziert eine Struktur, die in der Analyse des Schulhauses Breitenrain rekonstruiert wurde: Das Gebäude will gesehen werden und Bekanntheit erreichen. Wird dies beim Schulhaus Breitenrain durch die Positionalität an einem zentralen Verkehrsknotenpunkt erreicht, bedient sich das Schulhaus Hinter Gärten zwangsläufig anderer Mittel. Es wird in seiner Farbigkeit und Farbintensität zum Kunstwerk.

Wenn das Schulhaus diesen Anspruch einlösen kann, dann gewinnt es auch eine Ausstrahlung, die über die visuell wahrnehmbare Sichtweite hinausgeht. Denn als Kunstwerk wird es rezipiert und besucht, so wie man auch Museen aufsucht. ${ }^{42}$ Es kann festgehalten werden, dass das Gebäude eine Sendung in Anspruch nimmt. Die Sendung hat aber weniger die Funktionalität des Gebäudes zum Inhalt. Inhalt der Sendung ist die Architektur beziehungsweise die Farbe. Denn außer der auffälligen Farbgebung und der Größe des Gebäudes ist wenig bis nichts Auffälliges am Gebäude zu erkennen.

Mit der Farbgebung nimmt das Schulhaus einen Werkcharakter als ästhetisches Objekt für sich in Anspruch. Damit können zwei Lesarten gebildet werden. Zum Ersten kann es sich um eine Aufforderung handeln, das Gebäude als selbstgenügsames Werk zu betrachten. Zum Zweiten kann es als Repräsentation der 
Funktionalität gedeutet werden, was einen Verweis auf eine ästhetische Funktion des Gebäudes bedeutet.

Die Affinität zu einem Werk bekommt das Gebäude durch die Farbe und seine krisenevozierende ästhetische Wirkung in Form einer Erfahrungs- beziehungsweise einer Überraschungskrise. Das Gebäude drängt sich zwingend auf, da man sich der Suggestivität von Farben, egal ob sie naturgegeben oder künstlich sind, nur schwer entziehen kann. Dabei haben Farben einen vorprädizierenden Effekt. Die Farbwahrnehmung findet auf einer perzeptiven, vorbewussten Ebene statt und erzielt ihre Wirkung relativ unabhängig von den anderen visuellen Effekten (ausführlich Kandel 2012). Farbwahrnehmung bleibt ein unbewusster Wahrnehmungsvorgang, wenn er nicht ins Bewusstsein geholt und bewusst weiterverarbeitet wird. Deshalb haben Farben im Marketing einen hohen Stellenwert, wo sie mit spezifischen Gefühlslagen in Verbindung gebracht werden beziehungsweise positive Gefühle evozieren sollen. Der Bewusstseinsfähigkeit von Farbwirkungen sind aber Grenzen gesetzt. Beim Schulhaus Hinter Gärten kann sicher nicht von einer sublimen Wirkung der Farbe gesprochen werden. Das Gegenteil ist der Fall.

In der Betrachtung als Werk sind einige Punkte zu diskutieren. Kunstwerke werden jeweils doppelt hergestellt: einmal vom Künstler und einmal vom Rezipienten (vgl. Kapitel 3.1.9). Das Kunstwerk stellt eine zur Natur sublimierte Kultur oder, wie Oevermann es ausdrückt, eine „erstarrte Lebendigkeit“ (2000b: 459) dar. Um die erstarrten Sinnstrukturen zu Lebendigkeit zu erwecken, bedarf es einer zweckfreien Betrachtung in Muße. Als gelungenes Werk evoziert ein Werk eine Krise, für die es gleichzeitig eine mögliche Bearbeitung anbietet. Das Gebäude evoziert aus sich heraus eine Sinnlichkeit, die neue Erfahrungen und Erkenntnisse emergieren lässt, wenn sich die Betrachtenden darauf einlassen.

Wo knüpft das Gebäude an die Merkmale eines Kunstwerks an? Das Gebäude stellt in seinen monochromen und in seinen polychromen Farben in Kombination mit den unterschiedlichen Kubaturen ein abstraktes Gemälde dar. Die Fassaden sind schnörkellos und monochrom. Gestalterische Elemente sind neben der Farbe in dem Gefüge der Baukörper und in den Fensteranordnungen zu sehen. Die Gebäudeteile sind jeweils kontrastierend gewählt: hoch-niedrig, schmal-tief, lang-kurz. In der Komposition ergibt sich damit in der Seitenansicht ein Bild, wie es in der Tradition der neuen Sachlichkeit zu finden ist, zum Beispiel bei Camille Graeser, Max Bill, Hans Arp und vielen mehr. 
In der Architektur sind Farben ,ein ebenso kräftiges Mittel wie der Grundriss und der Schnitt. Oder besser: Die Polychromie ist ein Bestandteil des Grundrisses und des Schnittes selbst" (Le Corbusier, zit. nach Grütter 2015: 332). Diese Farbwirkung zeigt sich am Schulhaus Hinter Gärten explizit. Dabei zielt das Gebäude auf einen großflächigen monochromen Effekt in der Wechselwirkung mit der polychromen Gestaltung durch die Farbvarianz der Fassaden und der Gebäudeöffnungen. Die Farbigkeit der Innenräume stellen einen dritten polychromen Effekt dar, der nur ersichtlich ist, wenn das Gebäude von innen erleuchtet ist. Das Gebäude verkörpert einen wechselnden Rhythmus, der sich aus Farbwechseln und kubischen Gebäudevolumen zusammensetzt.

Vor der Deutung des Gebäudes als Werk ist zu fragen, inwieweit die Architektur Selbstzweck sein kann, also die Funktionalität dem Werkscharakter untergeordnet wird, oder inwieweit die krisenevozierende Wirkung die Funktionalität unterstützt. Denn Kunst und Pädagogik haben eine strukturelle Verwandtschaft in der Evokation eines Bildungsprozesses. Im Unterschied zur ästhetischen Bildung, welche der sinnlichen Präsenz des Werks und der Offenheit des Rezipienten in der Krise durch Muße bedarf, ist ein pädagogisch initiierter Bildungsprozess immer in irgendeine Form der pädagogischen Interaktion eingebunden. Die Kunstbetrachtung ist demgegenüber eine individuelle, subjektive, sinnliche und rein geistig-kognitive Angelegenheit. Die Schule Hinter Gärten repräsentiert mit der Selbstdarstellung eines Hauses als Kunstwerk eine gewisse Affinität zu der naturwüchsigen kindlichen Sinnlichkeit in der Weltwahrnehmung.

Die Deutung des Schulhauses als Kunstwerk lässt einen Unterricht oder eine Pädagogik erwarten, in denen die Sozialisanden sich in Muße primär selbst bilden und wo die Architektur einen Möglichkeitsraum emergiert, in dem sinnliches Erleben und kreative Aneignung gefördert wird.

Farben haben neben ihrem sinnlichen Werkscharakter auch eine Wirkung sozialer Attributierung: Sie fungieren als Zuschreibung sozialer Tatbestände. Die Verbindung von Farben mit Geschlecht, beispielsweise dass Mädchen Pink zugedacht wird, ergibt sich nicht aus der Qualität der Farbe, sondern aus sozialen Konventionen. Die Farbe ist ein Statusanzeiger, wie dies beispielsweise bei politischen Parteien üblich ist. Diese Statusanzeige gilt auch für Kinder. Kinder werden farbiger angezogen als Erwachsene, Kinderzimmer sind farbig möbliert usw. Komplett schwarz angezogene Babys oder Kinder sind eine offensichtliche Verletzung dieser sozialen Norm. Für Jugendliche gilt dieser Umstand gerade 
nicht mehr. Diese tragen oft uniform schwarze Kleider, gerade um sich von Kindern abzugrenzen und ihren Statusübergang zu repräsentieren. Auf Schulhäuser übertragen ist die Farbigkeit eine Statuszuschreibung und Teil sozialer Konventionen, welche die Gebäude als Bauten für Sozialisanden festschreiben. Dies gilt umso stärker, je kleiner die Kinder sind, für welche die Bildungsbauten errichtet sind. Kindergärten und Primarschulen sind in der Regel farbiger gestaltet als Gymnasien.

Für den Gegenstand Schulhäuser fallen in der Farbgebung mehrere Faktoren zusammen: Farbe wird mit ästhetischer Wahrnehmung, mit Kunst und Kreativität, aber auch mit Kindern in Verbindung gebracht. Als latenter Bedeutungsgehalt ist eine gewisse Affinität der beiden Lesarten zu konstatieren. Kindern und Kunst wird eine Verwandtschaft zugeschrieben, die darin rekonstruiert wurde, dass für Kinder die sinnliche krisenhafte Wahrnehmung der Welt den hauptsächlichen Modus der Welterschließung darstellt. Für kleine Kinder fällt die Krise durch Muße mit der Erfahrungskrise zusammen.

Die monochrome Farbgebung der Wände stellt die Farbqualität ins Zentrum des Erlebens. An den Fassaden wurden warme Farbtöne gewählt: Rostrot und Magenta. Trotzdem wirkt das Gebäude kalt, was dem Farbton diametral entgegensteht. Der Eindruck ergibt sich dadurch, dass das Gebäude den Selbstzweck als Kunstwerk in den Vordergrund stellt. Das Gebäude entzieht sich in seiner Gestik jeglicher autonomen Aneignung durch die Nutzer_innen. Es sind mehrere Merkmale und vor allem kleine Details, die in der nächsten Sequenz am Beispiel der Innen-Außen-Abgrenzung betrachtet werden.

Das Schulhaus Hinter Gärten ist unmittelbar an einer Straßenkreuzung platziert. Darüber hinaus befindet sich ,hinter' dem Haus auf der Südseite eine StraBe, die ein Zubringer zu dem Schuppen und/oder zu dem Acker zu sein scheint. Damit ist die Schule auf drei Seiten von Straßen umgeben, die den Gebäudekomplex abschließen. Dies wird durch die Farbgebung noch verstärkt: Die Bodenbeläge - ein Erschließungsweg im Westen und ein etwas größerer Platz im Nordosten - haben dieselbe Farbe wie die Gebäude. Dies markiert den Gebäudekomplex als Einheit in einem rechteckigen Raum, obwohl das Gebäudegefüge selbst kein Rechteck darstellt. Die Innen-Außen-Abgrenzung der Entität wird durch diesen Umstand hermetisch abgeriegelt. Sie fällt mit den Mauern des Gebäudes oder den Mauern der Umfriedung zusammen. Es gibt keine Übergangszone auf dem Perimeter des Gebäudes in Form von Gartenbeeten, einer Hecke 
oder eines Vorplatzes, wie dies beispielsweise beim Schulhaus Breitenrain festzustellen ist. Dort wo inselgleiche Bepflanzungen auszumachen sind - im Osten und andeutungsweise auch im Norden -, befinden sich diese auf dem Gehsteig.

Der Perimeter wird raumsparend der Funktionalität des Gebäudes als Binnenraum zugewiesen. Dabei ist die sparsame Raumnutzung nicht einem Platzmangel geschuldet: Der sparsame Umgang mit dem Raum scheint eher ein ,Programm‘ zu sein. Das Schulhaus ist raumökonomisch als urbanes Gebilde geplant, welches den vorhandenen Perimeter bis an die äußersten Grenzen ausreizt. Das Gebäude verkörpert damit einen durchrationalisierten ,Geist', der einen ziemlichen Kontrast zu den Häusern der Umgebung darstellt. Die Wohngebäude in der Gegend sind von Gärten umgeben. Die Häuser grenzen sich doppelt ab; einmal in einen privaten Außenraum und einmal innerhalb der zurückversetzten Gebäude. Das Schulhaus Hinter Gärten stellt in seiner Positionalität und der Innen-Außen-Abgrenzung eine konträre urbane Bedeutungsstruktur her: positioniert direkt an der Straße, an welcher wie ein Annex ein halb öffentlicher Raum angehängt ist.

Aus der Positionalität wurde rekonstruiert, dass sich das Haus hermetisch innerhalb des rostroten Rechtecks vom Außen abgrenzt. Diese Struktur reproduziert sich, wenn die Ausrichtung der Gebäude und die Erschließungen mitbetrachtet werden. Sowohl Fenster wie Türen sind gegen Norden und Süden ausgerichtet. Es gibt keine Zugänge aus dem Gebäude auf die seitliche Fläche. Im Gegenteil, die Fläche wird vom Gebäude nicht nur durch die Farbgebung, sondern zusätzlich durch eine unüberwindbare Doppelmauer abgegrenzt. Ein direkter Zugang aus dem Gebäude wäre einfach zu bewerkstelligen, indem Treppe und Rampe vertauscht würden. Das Gebäude verweigert sich einer Aneignung des Außenraums, indem es diesem explizit die ,kalte Schulter ${ }^{\star}$ zuwendet. Die Fläche scheint nicht zugehörig. Dass sie doch dazugehört, zeigt sich an den gestalterischen Elementen, die die Ausrichtung des Gebäudes aufnehmen. Die Fläche wirkt gestalterisch zugehörig und funktional abgegrenzt.

Für wen und für welche Tätigkeiten könnte das Gelände gedacht sein? Es sind einige Elemente, die den Platz als Park oder als einen Spielplatz auszeichnen (Abb. 17). Die Anordnung scheint wenig motiviert zu sein. Die Tische sind nachträglich und als mobiles Mobiliar dort platziert worden, wo gerade Platz vorhanden war. Abgesehen von einem unbespielten Sandkasten sind es Einrichtungen, auf denen balanciert werden kann. Insgesamt gibt es wenig Abwechs- 
lung. Die festen Installationen bestehen aus drei gleichartigen Teilen. Etwas vielseitiger sind die liegenden Baumstümpfe, die ebenso zum Balancieren gedacht sind. Ergänzt wird dieses Setting durch ein großes und ein paar kleinere runde Spielgeräte, deren Funktionen nicht eindeutig bestimmt werden können. Es werden vermutlich Artefakte sein, die zum Balancieren oder Rotieren konzipiert sind.

Das Gelände ist von heckenartigen Bepflanzungen durchsetzt. Das Gelände ist weder eindeutig Park noch Spielplatz; weder bepflanzter Garten noch nutzbare Fläche. Die Bepflanzungen sind äußerst steril. Die Artenvarianz ist klein, die Büsche quaderförmig zurechtgestutzt: Es herrscht Ordnung.

Als Schulhof kann dem Gelände kaum pädagogischer Wert zugeschrieben werden. Auf dem Gelände findet sich nichts Anschauliches, was einer lehrreichen Betrachtung oder Beobachtung z. B. in Biologie, Chemie oder Physik oder gar selbst durchgeführten Experimenten dienen könnte. Als Sportplatz oder für den Turnunterricht ist der Platz wiederum ungeeignet. Auch als Pausenhof bietet das Gelände wenig Anregung und ist nicht sehr einladend. Kurzum: Der Pausenhof wirkt ohne erkennbare Motivationen, Schulraum als Anregungsraum zu gestalten, sei dies für unterrichtliche oder nichtunterrichtliche Bildungsprozesse oder sonstige sinnliche Erfahrungen. Das Gestaltungsprinzip der Grünanlage ist mehr auf eine ästhetische Unterstützung des Gebäudes gerichtet, als dass es ein eigenlogisches Gebilde darstellt, das als Spielplatz, als Park für gemeinschaftliche Tätigkeiten und dergleichen konzipiert ist. Aber auch der Funktion als ästhetische Unterstützung sind enge Grenzen gesetzt. Die Fläche konfligiert mit der Logik des architektonischen Gefüges. Das Gebäude grenzt sich innerhalb des roten Rechtecks ab und präsentiert sich als urbanes Gebäude. Das zur Seite gestellte parkähnliche Gebilde konterkariert diese ,naturlose ' Bedeutungsstruktur. Die Fläche scheint unmotiviert und ist wohl dem Zwang eines Bauprogrammes geschuldet, in welchem eine Grünfläche zwingend festgeschrieben ist, ohne dass dieser eine wesentliche Funktion zugedacht wird.

Ein Sachverhalt soll an dieser Stelle geklärt werden. Bisher wurde selbstverständlich und unhinterfragt vom Schulhaus Hinter Gärten gesprochen, unabhängig davon, ob das Gebäude als Schulhaus identifiziert werden kann. Es ist zu fragen, was dieses Schulhaus zu einem Schulhaus macht. 


\section{Exkurs: Strukturprinzipien des Gebäudetyps Schulhaus}

Es ist erstaunlich, wie sehr öffentliche Schulhäuser einen eigenen, unverwechselbaren Gebäudetyp darstellen. Wie sonst ist zu erklären, dass Schulhäuser unmittelbar innerhalb von Sekundenbruchteilen eindeutig als Schulbauten erkannt werden? Dies ist unabhängig davon der Fall, ob es sich um Schulhäuser der Primarstufe oder um Gymnasien handelt, egal, ob es sich um große, monumentale Schulkasernen handelt oder um Pavillonschulhäuser oder kleine Dorfschulhäuser. Auch die historische Epoche und der Baustil der Erstellung spielt faktisch keine Rolle: Schulbauten werden als solche intuitiv, unvermittelt und eindeutig erkannt. Dabei sind es keine angebrachten Embleme oder Schriftzüge, die Schulen als solche kennzeichnen. Den Schulhäusern sind latente sinnhafte Strukturprinzipien eingeschrieben, die das (Wieder-)Erkennen als Schulen garantieren. Dieser Umstand zeigt sich insbesondere daran, dass sie auch in unterschiedlichen Bildungssystemen in den Kantonen oder über die nationalen Grenzen hinaus ähnlich strukturiert sind. Der moderne Nationalstaat bringt in sozialhistorisch-kulturell unterschiedlichen Gesellschaften Schulbauten hervor, die nach ähnlichen Strukturprinzipien und Mustern gestrickt sind (vgl. Caruso 2003; Châtelet 1999; Göhlich 1993; Schneeberger 2005). Diesen Umstand kann man sich daran klarmachen, dass er für Privatschulen gerade nicht gilt. Diese sind mit Ausnahme z. B. der Waldorfschulen mit ihren eigenen ageometrischen Architekturen - oft in Räumen einquartiert, die für andere Zwecke erbaut wurden, und sie sind von außen dementsprechend meistens nicht als Schulen erkennbar.

Es kann also konstatiert werden, dass Bauten der öffentlich-rechtlichen Schule die Schule - oder wie bereits rekonstruiert wurde, u. U. vielmehr auch den Staat - in einer unverwechselbaren Art und Weise repräsentieren. Da dies ungeachtet ihrer konkreten einzelfallspezifischen Materialisierung der Fall ist, kann im Weiteren festgehalten werden, dass in den Architekturen dieser Bauten objektiv vorhandene und auf einer perzeptiv-vorbewussten Stufe erfahrbare Strukturprinzipien versteinert vorliegen, deren Sinngehalte über eine lange Periode hinweg dauerhaft geteilt werden.

Welche manifesten und latenten, sinnhaften Strukturprinzipien sind Schulbauten gemein, dass sie einen eindeutigen Gebäudetyp charakterisieren? Wie kommt es, dass der Auftrag des Gebäudes - die Beherbergung und damit der Betrieb einer Schule - architektonisch so umgesetzt wird, „als ob [er] in einer 
geheimen Zentralisierung erstellt worden sei (...)“(Knutti-Baumann 1997: 358)? Diese «geheime Zentralisierung» ist umso erstaunlicher, wenn die jeweils zeitgenössischen Diskurse mitbetrachtet werden. Denn seit es Schulbauten gibt, werden deren Unzulänglichkeiten von pädagogischer Seite her angeprangert (beispielhaft Gonzenbach et al. 1933; Kollmann 1890; Montag Stiftung Jugend und Gesellschaft Bonn 2011; Nötzli 1894).

Es sind verschiedene Strukturmerkmale hervorzuheben, die für ein öffentlich-staatliches Schulgebäude ,typisch“ sind. Die Merkmale wurden an den verschiedenen Schulhäusern rekonstruiert. ${ }^{43}$ Sie werden in abkürzender Weise hier in generalisierter Form wiedergegeben.

$\mathrm{Zu}$ unterscheiden sind zunächst deskriptive Strukturmerkmale: Kennzeichnend für Schulbauten ist das spezifische Verhältnis von unbebauter zu bebauter Fläche. Meist ist nur ein kleiner Teil der Fläche mit Gebäuden bebaut. Großen Anteil haben unbebaute Flächen. Weiter sind es Strukturmerkmale, die das spezifische Ensemble von Funktionsbauten und -feldern aufweisen.

Die unbebaute Fläche ist bei Schulgebäuden in funktional differenzierte Felder unterteilt: Sportfelder, Rasen- und Hartplätze, Spielplätze, überdeckte Pausenhallen, freie Flächen, bepflanzte Flächen, Erschließungswege usw. Allen voran stehen die Sportfelder in Verbindung mit großen Häusern unverkennbar für eine Schule. Sportliche Betätigung als körperliche Ertüchtigung und Installationen für leibbasierte Erfahrungen und Tätigkeiten, wie Schaukeln, Balancieren oder Anlagen der Leichtathletik, nehmen auf Schulplätzen einen zentralen Stellenwert ein. Auf Schulanlagen können bis zu 50 unterschiedliche konstruierte Artefakte ausgemacht werden, die jeweils für sich eine Einübung einer spezifischen Bewegung oder Bewegungsabläufen darstellen.

Die dritte Gruppe von Strukturmerkmalen betrifft die Gestaltung des Geländes und der Bauten: Schulbauten sind auch an ihren Proportionen zu erkennen. Ab der Mitte des 19. Jahrhunderts werden vor allem städtische Schulhäuser für eine große Zahl von Schüler_innen errichtet. Charakteristisch für Schulbauten der Primarstufe ist, dass die Höhe der Gebäude limitiert ist. Im Gegensatz zu anderen öffentlichen Gebäuden wie Spitäler, die beliebig hoch gebaut werden 
können, sind Schulbauten selten mehr als drei-, oft eher zweistöckig angelegt. Dies liegt an der Aufsichtsproblematik und wird durch die einheitlich zeitliche Rhythmisierung des Unterrichts verschärft. Eine große Zahl an wilden und ungestümen Schüler_innen drängt zeitgleich durch Korridore, Treppen und Türen. Die undisziplinierten Verhaltensweisen von Kindern muss als Problematik von der Architektur aufgenommen und so weit wie möglich entschärft werden.

Was Schulbauten im Weiteren auszeichnet, ist die serielle Raumgestaltung. Schulen sind typischerweise als Massenveranstaltung geplant, wodurch viele gleichartige Unterrichtsräume vorhanden sind. Ein weiteres Strukturelement von Schulgebäuden ist die Farbigkeit von Architektur. Beim Gebäudetyp Schulhaus werden fast ausnahmslos Farben als Gestaltungsmittel in einer ganz eigenen schulspezifischen Art und Weise eingesetzt, wie dies für keine anderen Bauten der Fall ist. Es werden oft bunte Akzente gesetzt, die im Kontrast zur Monumentalität der Gebäude stehen.

Zusätzlich gibt es einige wenige Strukturmerkmale, die geradezu als Insignien oder Embleme von Schulhäusern gelten können. In ihrer Summe ist es erstaunlich, wie wenige Strukturmerkmale dafür verantwortlich sind, dass wir ein Schulgebäude als solches erkennen. Am offensichtlichsten wird dies, wenn Darstellungen von Schulbauten betrachtet werden, die auf diese Strukturprinzipien reduziert sind: zum Beispiel in Cartoons und Zeichnungen in Kinderbüchern. Als unverkennbares Merkmal eines Schulhauses finden sich fast ausnahmslos ein stilisierter Turm, eine Uhr, eine Glocke und das Hochparterre. Dabei sind Uhr und Turm häufig so angebracht, dass sie keine funktionale, sondern eine rein symbolische Wirkung entfalten (vgl. die Interpretation des Schulhauses Breitenrain; Kap. 4.4). Diese Embleme teilen sich Schulhäuser auch mit anderen Gebäudetypen, z. B. mit Kirchen. Es kommen also weitere Prinzipien wie serielle Räumlichkeiten und eine gewisse Rationalität in den Bedeutungsstrukturen dazu.

Bei der Analyse des Schulhauses Hinter Gärten lässt sich - abgesehen von der Farbe und der seriellen Raumordnung - keines der explizierten Strukturmerkmale erkennen. 


\subsubsection{Das Verschwinden der Schule}

Auf der rechteckigen Grundfläche erheben sich zwei Gebäudevolumen: ein länglicher dreigeschossiger Trakt mit sieben Fensterachsen. Nördlich daran angebaut ist ein tiefer gelegenes Gebäudevolumen, das den Eindruck einer großen Halle erweckt. An der Straßenkreuzung gelegen ist eine quadratische Außenfläche, die tiefer gesetzt ist. Das verleiht ihr mehr den Charakter eines Hofs als denjenigen eines Vorplatzes.

Die zwei Teile verweisen auf zwei unterschiedliche Funktionen, die den Auftrag des Gebäudes ausmachen. Der längliche Trakt suggeriert eine geistige, wissensbasierte Tätigkeit, während die Halle auf ein Produktionsgebäude hinweist. Das Gebäude strahlt eine posttayloristische Trennung von Produktion in der Fertigungshalle einerseits sowie Entwicklung und Verwaltung im Längstrakt andererseits aus. Die Proportionen sind so aufgeteilt, dass - in diesem Vergleich - relativ viel Entwicklungs-, Forschungs- oder Dienstleistungsarbeit im Verhältnis zur Produktion notwendig ist: Der Bau verweist auf hochspezialisierte ,Produkte' mit einem hohen Anteil an geistigen Tätigkeiten, ähnlich wie in der Hightech-Industrie oder einer Produktionsstätte im Sinne einer ,Industrie 4.0", die eine Verzahnung von Fertigung, Kommunikations- und Informationstechnologien und einer Produktion in time vorsieht. Lagerhallen sind keine auszumachen, genauso wenig wie Zufahrtswege. Von der Gebäudeaufteilung her wäre es auch als ein Spital oder Pflegeheim denkbar, bei denen ein Wohn- beziehungsweise Bettentrakt und ein Trakt mit Ambulatorium, Aufenthaltsräumen, Cafeteria und Ähnlichem räumlich getrennt wird. Den Gebäuden wird eine zweigeteilte Funktionalität zugewiesen. Die Gebäudeformen scheinen dabei nüchtern und zugleich uneindeutig, sie lassen keine eindeutigen Schlüsse auf die Funktionalität zu.

Auffallend ist der (Vor-)Platz, der eine gewisse Repräsentativität darstellt. Außeralltäglich ist dabei vor allem die Farbe. Auch sie lässt Assoziationen an ein Hightech-Unternehmen aufkommen. Ohne die Farbgebung kann man sich das Gebäude sehr gut als einen gesichtslosen, dienstleistungslastigen Industriebau imaginieren.

Im Folgenden werden die Fassaden und Eingangsbereiche betrachtet, die die Eröffnungsprozeduren eines Gebäudes darstellen. Sie sind die einladende oder abweisende ,Begrüßung` eines Gebäudes. Das Gebäude Hinter Gärten wird 
durch die Farbmarkierung in seinem Rechteck relativ hermetisch abgeriegelt. Zusätzlich haben die Doppelmauern eine abweisende Intention. Denn von der Seite her sind sie eine fast unüberwindbare Hürde (Abb. 16, 22). Sie stellen funktional die Geländer einer Rampe dar, welche von der Treppe getrennt ist. Damit haben die Mauern und die Rampe mehrfache, aber auch konträre Funktionen.

Die Mauern sind Einfriedung und verhindern das Betreten des Geländes. Die Rampe als Teil von hindernisfreiem Bauen stellt den Zugang für Rollstuhlfahrende sicher. Die Rampe, die eigentlich die Zugänglichkeit des Hauses für Rollstuhlfahrende erhöht, wirkt durch die Umsetzung mit der Doppelmauer eher wie eine Abkapselung und Verschließung des Gebäudes. Die Wirkung zwischen intendierter Öffnung des Gebäudes und der damit objektiv hergestellten Schranke ist paradox.

Die Intention einer Rampe ist das Befahren. Gerade für Kinder stellen Rampen eine fast zwangsläufige Handlungsaufforderung dar, hinunterzurennen oder mit allerlei Fahrzeugen runterzufahren. Die Anordnung der Doppelmauer verhindert genau dieses durch äußerst sublime bis perfide Art: Die Enge der Rampe und der grobe Verputz machen es unmöglich oder zumindest gefährlich, die Rampe zu befahren oder auch hinunterzurennen. Die Mauern sind so platziert, dass die Verletzungsgefahr an dem groben Verputz groß ist. Sie erzwingen ein diszipliniertes Gehen.

Wenn der Schulhausplatz als Ort für Kinder zum Spielen gedacht ist, ist die Rampe dysfunktional. Zumindest dann, wenn man das Potenzial einer Rampe in einer größtmöglichen Vielfalt von (spielerischen) Nutzungen sieht. Barrierefreies Bauen könnte technisch problemlos so umgesetzt werden, dass sich daraus unterschiedliche Nutzungsmöglichkeiten ergeben. In der konkreten Umsetzung am Schulhaus Hinter Gärten ist die Rampe genau so arrangiert, dass sie monofunktional den einen konzipierten Zweck erlaubt und alle anderen Aneignungen unterbindet. Jegliche Performanz, die nicht explizit durch die Architektur intendiert ist, wird verhindert. Die explizierte Sinnstruktur reproduziert sich an der Doppelmauer sowohl funktional als auch symbolisch. Ein hindernisfreier Zugang kann so gestaltet werden, dass verschiedene Nutzungen und Tätigkeiten eröffnet werden z. B. auch das ,Bespielen“ und ,Befahren“. Mauern können so gebaut werden, dass sie auch als einladende Sitzgelegenheiten oder Ähnliches fungieren. 
Dies ist insofern von besonderem Interesse, als sich Kinder räumliche Settings und Gegenstände in der Regel anders aneignen, als dies Erwachsene tun. Erwachsene nutzen die räumlichen Gegebenheiten gemäß den vorstrukturierten Zweckbestimmungen. Dinge und Räume haben einen spezifischen Gebrauch. So gehen Erwachsene auf den zum Gehen vorgesehenen Pfaden, während Kinder fast ausnahmslos die Randpflasterungen nutzen; sie gehen auf den Mauern oder Geländern, sie springen auf dem Fußgängerstreifen von Streifen zu Streifen usw. Ihre Aneignungen sind oft geradezu invers zur gebauten Zweckbestimmung (siehe Muchow \& Muchow 2012: 106 ff.). Unter dieser Betrachtung erhält die Rampe einen erzieherischen oder sozialisatorischen Gehalt in der Evozierung von Affektkontrolle und einer Disziplinierung des Verhaltens. Zum Schutz der körperlichen Unversehrtheit müssen die Kinder die affektive Intention zügeln, mit einem Skatebord oder Fahrrad die Rampe hinunterzufahren. Die latente Sinnstruktur bezüglich eines sozialisatorischen Effektes dieser Rampe ist also folglich, dass Affektkontrolle und eine Gefolgschaft gegenüber den vorstrukturierten Handlungsvollzügen durch Dinge einsozialisiert wird.

Bemerkenswert scheint mir, dass sich in unscheinbaren architektonischen Artefakten eine erzieherische Funktion der Schule objektiviert, die insbesondere in der Disziplinierung der Schüler_innen über Affektkontrolle liegt.

Die Rampe führt hinunter auf einen Platz, der dieselbe Farbe wie die Gebäudefassaden hat, was ein ganz spezifisch sinnliches Farberleben auslöst. Er ist primär leer, entfaltet seine Wirkung ausschließlich durch die monochrome Farbe. Auf den Detailfotografien sind bei näherer Betrachtung einzelne Möblierungen erkennbar. Diese sind jedoch so gestaltet und platziert, dass sie weitgehend unsichtbar bleiben und auch nicht zur Nutzung einladen: eine Sitzgelegenheit unter dem Dach, zwei Basketballkörbe am äußersten Rand des Geländes. Wenn schon die Präsenz alltäglicher Gegenstände als ein potenzieller Störfaktor angesehen werden, lässt sich eine weitreichendere Folgerung festmachen: Wer sich auf dem Hof aufhält, wird selbst zum ,Störfaktor ‘. Zumindest wird man sich gewahr werden, dass man auf dem monochromen Hintergrund des Bodenbelags und Gebäudes besonders auffällt. Man gerät zwangsläufig in den Aufmerksamkeitsfokus 
und steht im Rampenlicht. ${ }^{44}$ Die ästhetischen Komponenten des Gebäudes erwecken tendenziell die Assoziation, dass nicht nur Gegenstände, sondern auch die eigene Person als störend betrachtet wird.

\subsubsection{Spiel ohne konstitutive Regeln}

Die einzigen Objekte, die explizit zu einer Tätigkeit einladen, sind zwei Basketballkörbe (Abb. 18). Die Körbe stehen nebeneinander. Damit ist die Platzierung der Körbe eine Verletzung der konstitutiven Regeln des Basketballspiels, bei welchem auf einem Basketballfeld zwei Mannschaften bestehend aus je fünf Spielern auf die je gegenüberliegenden Basketballkörbe spielen. Auch die Absenz von Bodenmarkierungen verweisen darauf, dass die Körbe nicht für die Durchführung eines Matches bestimmt sind. Die Position der Körbe entspricht zweier nebeneinanderplatzierter Fußballtore. Eine Anbringung der Körbe, welche ein Spiel erlauben, wäre aber durchaus möglich. Die Platzierung der Körbe dient also voraussichtlich dem Üben des Korbwurfs und nicht einem Spiel. Allenfalls können sie für eine Variation des Basketballs, den Streetball, benutzt werden, bei dem je ein Spieler gegen einen anderen antritt.

Die Positionierung der Basketballkörbe lässt den Schluss zu, dass sie für individuelle Tätigkeiten und nicht für eine gemeinsame Tätigkeit einer Gruppe gedacht sind. Die Platzierung verkörpert und versinnbildlicht einen latenten Sinn, in dem nicht das regelgeleitete Spiel das Entscheidende ist. Die Sinnstruktur ist das Üben eines Wurfes. Über das immer gleiche Werfen von Bällen werden die individuellen skills verbessert. Das Ziel ist die Routinisierung singulärer Techniken durch Training. Es geht um einen zukünftigen, im Ernstfall liegenden (Score-)Erfolg, der eine gegenwärtige Übung durch immerwährende Routine bedarf. Dabei liegt der Erfolg darin, einen Korb zu treffen, jede und jeder für sich, unabhängig von einer Mannschaft. Der Erfolg liegt einseitig auf dem ScoreErfolg. Es gibt keine Rollen, zum Beispiel als Center oder als Guards, die außer-

44 Der physikalische Grund dafür ist, dass die Wahrnehmung der äußeren Erscheinung eines Objektes im Wesentlichen vom Kontrast des Objektes zu seinem Hintergrund abhängt. Auf monochromem Hintergrund fällt jedes Objekt besonders auf, das in einer anderen Farbe gehalten ist (Kandel 2012: 299). 
halb des Treffers liegen. Es gibt keinen Erfolg etwa in Form gelungener Spielzüge. Als Bedeutungsstruktur wird eine individualisierte Tätigkeit außerhalb von konstitutiven Regeln des Spiels repräsentiert und intendiert, in welcher nur der eigentliche Treffer das erstrebenswerte Ergebnis ist.

Ergänzt werden soll, dass die beiden Körbe auch für Übungszwecke gänzlich unpraktisch montiert sind. Unpräzise Bälle landen außerhalb des Areals auf der Straße, was sowohl für die Sicherheit der Schüler_innen als auch der Verkehrsteilnehmenden problematisch ist. Mit einer Anbringung auf der gegenüberliegenden Seite hätte man dies technisch einfach verhindern können. Es hätte aber die Repräsentationswirkung des Gebäudes transformiert. Aufgrund der Farbe könnte das Gebäude dann sehr gut mit dem Sitz einer Basketballvereinigung verwechselt werden. Diese Überlegung verweist erneut darauf, dass die Körbe nicht wirklich als Funktionsgegenstände gedacht sind. Als Sitz eines Basketballclubs wären sie als Repräsentationsobjekte beziehungsweise Embleme vorstellbar und sinnvoll. Für das Haus Hinter Gärten scheinen sie - genau wie die Grünfläche - einem Kompromiss entsprungen zu sein, der den Zwängen durch Vorgaben geschuldet ist, die Architektur in ihrer Bedeutungsstruktur aber eigentlich konterkarieren. Sie sind so weit entfernt wie möglich angebracht, wo sie die sinnliche Suggestion des Gebäudes am wenigsten stören.

Wie können die Basketballkörbe interpretiert werden? Unter einer funktionalen Betrachtung sind sie für einen Schulhausplatz als singuläre Spielgelegenheit eher ungeeignet. Es bleibt der Eindruck, dass Elemente lebendiger Aneignung im Gesamtensemble wie ein Störfaktor wirken.

Das Schulhaus hat eine Gestik, die auf die Verhinderung von Aneignung, Tätigkeiten und Performanz gerichtet ist, da diese den Werkcharakter beeinträchtigen. Die singuläre und keinesfalls repräsentative Beobachtung bei meinem Besuch des Schulhauses weist auf diese abweisende Wirkung hin. Das Schulhaus Hinter Gärten war das einzige Schulhaus, wo ich keine Kinder auf dem Schulhof angetroffen habe. Gegenüber auf dem Gelände des Kindergartens war eine Gruppe von Kindern am Fußballspielen. Dies kann als Verweis gedeutet werden, dass das Haus von seinen Nutzer_innen wenig angenommen wird.

Die Wirkung eines hermetisch verschlossenen Gebäudes äußert sich auch durch die Lage der Gebäudekörper und Fenster. Die Fenster des hohen, langen Hauptgebäudes sind an der Nord- und Südseite angebracht. Die Ostseite, die am nächsten zur Öffentlichkeit liegt, präsentiert sich als eine monotone, fensterlose 
Mauer. Die abweisende Gestik der Doppelmauer an der Nordseite reproduziert sich. Umgekehrt muss festgehalten werden, dass damit die größtmögliche Fläche und Wirkung eines monochromen Gemäldes auf der Seite der Öffentlichkeit hergestellt wird. Die Öffnungen an der Nordseite sind, wie das ganze Gebäude, von der Straße zurückversetzt. Die Fenster fungieren daher vor allem als Ausblicke und nicht als Einblicke. Die ,Durchlässigkeit" durch die Transparenz ist eindimensional. Als Einblick erhält man, sofern im Inneren des Gebäudes künstliches Licht brennt, einen Farbkontrast durch die Farbigkeit der Räume.

Eine ähnliche Struktur zeigt sich an der Südseite des Gebäudes. Die großen Fenster sind als Ausblicke aufs freie Feld konzipiert. Das Gebäude ist so platziert und ausgerichtet, dass es von außen her abgeschottet ist, von innen heraus aber Ausblicke insbesondere nach Süden auf die unbebaute Brache bietet.

Zusammenfassend kann festgehalten werden, dass das Gebäude in seiner Form einen äußerst sparsamen, fast spartanischen Charakter hat. Es repräsentiert eine Funktionalität, die auf eine durchrationalisierte dienstleistungsorientierte Ökonomie gerichtet ist. Über seine ästhetische Gestaltung nimmt es nach außen eine Sendung in Anspruch und kapselt sich zugleich ab: Das Gebäude drängt seine expressive Wirkung zwangsläufig und fast exzessiv ins Bewusstsein der Vorübergehenden. Gleichzeitig entzieht es sich jeglichen Aneignungen, da diese die Wirkung des Gebäudes stören.

Die Kombination von Schlichtheit in der Form und opulenter Farbgebung stellt eine ambivalente Atmosphäre her. In der Interpretation als Schulhaus präsentiert das Gebäude ein Bildungsideal einer asketischen, in sich gekehrten Kontemplation. Bildungsprozesse vollziehen sich eindimensional beim Rezipienten: Sie werden extern ausgelöst, finden aber im Inneren der Subjekte statt.

Es wurde schon einiges über die Funktion der Erschließung gesagt, dies soll in der folgenden Sequenz vertieft werden.

\subsubsection{Die Schule als begehbares Werk}

Das Gebäude verfügt über drei Zugänge zu zwei Türen, die beide im niedrigen Trakt und nach Norden ausgerichtet sind. Beide Türen sind von außen nicht ersichtlich. Aufgrund der Lage des Gebäudes und des Vorplatzes kann kein Eingang eindeutig als Haupteingang bestimmt werden. Die westliche Erschließung 
führt direkt auf den Gehsteig. Der östliche Eingang hingegen wirkt durch den hofartigen Vorplatz repräsentativer. Es scheint, dass je ein Eingang zu einem der beiden Gebäude führt: Der westliche Eingang stellt den Zugang zum hohen Gebäude dar und der östliche Eingang denjenigen zur niedrigen Halle. Unmotiviert wirkt der Zugang von Süden her, da dieser ins Nirgendwo führt. Als alternative Lesart muss davon ausgegangen werden, dass auf dem Feld eine Überbauung geplant ist. Doch soll zunächst der westliche Eingangsbereich betrachtet werden (Abb. 15, 19).

Das tiefer gelegene Gebäude hat nicht nur einen direkten Zugang, sondern es erlaubt auch Einblicke. In der Fassade sind fünf Öffnungen eingelassen, wovon eine ein Durchgang ist. Sowohl die großen Fenster als auch der Eingang vom Gehsteig sind durch einen Niveauunterschied abgesetzt. Eine Rampe führt zur Maueröffnung, was den Einblick durch die Fenster verhindert. Der Eingangsbereich ist so angelegt, dass man von Osten kommend frühzeitig den Weg einschlagen muss, der zur Öffnung führt. Auch die Fenster gewähren den Vorbeigehenden nur bedingt einen Einblick ins Innere. Es ist nicht möglich, direkt entlang der Wand zu gehen, um einen Blick ins Gebäude zu werfen. Es muss explizit ein Umweg in Kauf genommen werden. Das barrierefreie Bauen ist auch hier so umgesetzt, dass ein direkter Zugang nicht mehr gegeben ist, obwohl dies mit zwei Tritten frontal vor dem Eingangsbereich einfach zu bewerkstelligen wäre. Vorbeikommenden wird der Blick durch die Fenster ins Innere zumindest teilweise verstellt und ein Zutritt erschwert. In der Konsequenz wird das Gebäude eindeutig als Gebäude für die Nutzer_innen markiert.

Die Fenster sind transparent; sie intendieren Ein- und Aussichten. Die Blickrichtung der Fenster des Hallentrakts ist im Prinzip umgekehrt zu derjenigen des hohen Gebäudes, welches Ausblicke, aber keine Einblicke erlaubt. Die Fenster der Turnhalle erlauben zumindest theoretisch Einblicke, aber keine Ausblicke.

Wenig einladend wirkt auch der höhlenartige, dunkele Eingangsbereich. Wobei sich hier wiederum ein überraschendes Moment einstellt: Wer den Eingangsbereich betritt, dem eröffnen sich neue Einblicke und erneut ein krisenhaft evoziertes Farberlebnis (Abb. 19). Entgegen der erwarteten Düsternis wird ein Farbenmeer betreten. Das monochrome Bild transformiert sich zu einer polychromen Skulptur. Das eröffnete Raumbild ist durch zwei Merkmale geprägt: Auf der einen Seite werden transparente Durchsichten in verschiedene Räume 
und durch Räume hindurch gewährt. Zugleich sind diese Räume in unterschiedlichen, je monochromen grellen Farben gehalten. Betrachten wir dies der Reihe nach.

Mit dem überdachten Bereich wird die Pausenhalle betreten. Die Pausenhalle ist in einem satten Himmelblau gestrichen, wobei Wände und Decke im selben Farbton gehalten sind. Der Boden und die Seiten der säulenartigen Tragestrukturen haben denselben Farbton wie die Außenfassade. Der Raum führt zu einer Glastür, die in einen grellgrün gestrichenen Raum führt. Auf der linken Seite befinden sich drei große Fenster, die auf Kniehöhe beginnen. Sie erlauben einen Blick in die Turnhalle, die gelb ist. Auf der rechten Seite sind zwei Einlassungen vorhanden, die als Zugang von Süden fungieren. Die Ein- und Durchblicke zeigen, dass jeder Raum eine andere Farbe hat: Gelb die Turnhalle, Grün die Eingangshalle, und durch die Turnhalle ist ein Gang ersichtlich, der himmelblau wie die Pausenhalle ist.

Die Erschließung führt durch einen Raum, der höhlenartig, von außen düster und wenig einladend wirkt. Eine einzige Sitzgelegenheit ist an einem unwirtlichen Ort im Durchzug platziert, insgesamt erscheint der Raum äußerst karg. Die Fenstereinlassungen können als Sitzbänke benutzt werden, obwohl sie nicht explizit als solche markiert sind. Die Gestik des Fensters und die Sitzrichtung der eingelassenen Bänke sind widersprüchlich: Die Fenster laden zum Blick in die Halle; wer sich hinsetzt, hat die Halle im Rücken.

Im Hinblick auf die Bezeichnung als ,Pausenhalle“ drängt sich die Frage auf, was hier in den Pausen gemacht wird. Es sind keinerlei Möblierungen oder Anregungen vorhanden, die zum Verweilen oder zu Tätigkeiten einladen. Einzig die Farbe ,hellt' diesen eher unwirtlichen Durchgangsraum auf. Die erkennbaren Intentionen des Raums sind passiv: Zum einen wird der Blick in die Turnhalle eröffnet, zum anderen könnte man sich der kontemplativen Betrachtung des durch die Architektur entstehenden Werks widmen. Die Pausenhalle adressiert die Schüler_innen als Zuschauer oder kontemplative Rezipienten. Die durch Architektur angeregte Tätigkeit im Nichtunterricht ist das Betrachten anderer 
beim Turnen. ${ }^{45}$ Die Transparenz, die sich durch die großen Einlassungen in den Mauern herstellt, dient damit kaum einer funktionalen Transparenz des Zuschauens, sondern in erster Linie der Konstituierung der ästhetischen Wirkung, durch die Verschiedenfarbigkeit der Räume. Der Farbeindruck wird durch die Sättigung bis zur Grellheit der Farben verstärkt, die Räume werden geradezu zum Leuchten gebracht. Bevor dieser Umstand weiter erörtert wird, soll zunächst der zweite Eingang betrachtet werden.

Zur Straße hin ist dieser durch eine Mauer, nordseitig mit einer Doppelmauer abgegrenzt. Wo die Mauer unterbrochen ist, ist der Bürgersteig mit einer bepflanzten Inselrabatte unterteilt. Es entsteht damit ein äußerer und ein innerer Teil des Bürgersteigs. Man muss - analog zum westseitigen Eingang - bereits früh den richtigen Weg einschlagen, um den Treppenansatz zu erreichen. Die Abgrenzung, wo der Bereich des Gebäudes beginnt, ist durch die Mauern und die Farbgebung explizit und trennscharf markiert. Um zu dem Gebäude zu gelangen, muss eine Treppe hinuntergestiegen und der hofartige Platz überquert werden. Die Treppe ist so platziert, dass der Hof diagonal gequert, also der größtmögliche Weg zurückgelegt werden muss. Kürzer wäre eine Erschließung entlang der Gebäude. Die Annäherung in der Diagonalen hat zur Folge, dass man sich nicht ungesehen dem Eingang nähern kann. Die Farbgebung des Platzes unterstützt diesen Umstand. Sie hat eine Wirkung wie der Lichtkegel des Bühnenlichts: Bei der Querung des Platzes steht man automatisch im Rampenlicht. Befindet man sich auf dem Hof, blickt man durch drei Öffnungen in einen überdeckten Raum, der spiegelverkehrt analog zum Raum an der Ostseite gestaltet ist.

Insgesamt sind die Zugänge gleichartig gehalten. Doch im Gegensatz zur ersten Assoziation führen die beiden Zugänge nicht zu unterschiedlichen Gebäuden. Als Gemeinsamkeiten ist die gesteigerte Krisenevokation durch die Farbe zu nennen. Der Überraschungseffekt kommt dadurch zustande, dass die Pausenhallen als dunkle, höhlenartige Räume wirken. Der Blick ins Innere öffnet sich erst beim Betreten dieser Vorbereiche. Die Atmosphäre der Pausenhalle weckt einen künstlerischen und auch affirmativen Wert des Wohlgefallens. Für den

Wobei man am Rande erwähnen kann, dass in den Pausen die Schüler_innen, die Turnunterricht haben, noch oder schon wieder in den Garderoben sind. Die Turnhalle wird dadurch für potenzielle Betrachter oft leer sein und ist allenfalls als Farbraum interessant. 
Besucher stellt sich ein krisenhaftes Staunen ein. Wiederum steht hier die künstlerisch-ästhetische Komponente im Zentrum.

Es wurde bereits festgestellt, dass das Schulhaus Hinter Gärten nicht intuitiv als Schulhaus erkannt wird, da weder entsprechende Embleme noch die spezifische Typik eines Schulhauses vorhanden sind. Auch Sportplätze und Spielgelegenheiten fehlen oder erscheinen nicht wirklich zugehörig.

Die Pausenhallen eröffnen nun den Blick auf die Sportstätte. Der Schulsport ist nach innen und unter die Erde verlagert. Assoziativ-metaphorisch wird der Schulsport ins Unbewusste des Gebäudes verlegt. Doch ist die Turnhalle vor dem eigentlichen Schulgebäude platziert. Um ins Schulgebäude zu gelangen, muss sie zwangsläufig passiert werden. In der Reihenfolge der Raumanordnung wird dem Sportunterricht also wie beim Schulhaus Breitenrain eine zentrale Funktion eingeräumt.

Im Vergleich zur Fallrekonstruktion des Schulhauses Breitenrain können aber ganz zentrale Unterschiede festgehalten werden. In erster Linie sind dies Fragen der Zugänglichkeit. Die Sporthallen sind nicht mehr im Pausenhof beziehungsweise Freizeitbereich angesiedelt, wo sie den Schüler_innen und den Kindern des Quartiers in Zeiten des Nichtunterrichts zugänglich sind. Dies gilt auch für die Turnhalle beim Schulhaus Breitenrain. Der Unterschied liegt darin, dass zusätzliche Sportplätze und Spielgelegenheiten vorhanden sind.

Beim Schulhaus Hinter Gärten sind die Turnhallen so platziert, dass von den Pausenhallen der Blick stets auf die Sportanlage fällt. Sie sind aber nicht mehr ein Raum, der für autonome Tätigkeiten verfügbar ist, egal ob diese in der intendierten sportlich-spielerischen Natur liegen oder ob sie als Aufenthaltsräume zu nichtintendierten Zwecken genutzt werden. Sportliche und spielerische Betätigungen finden ausschließlich in Form von organisierten Bildungsveranstaltungen im Unterricht statt. Darüber hinaus stehen sie für nichtschulische Zwecke zur Verfügung. Sie werden jedoch lediglich organisierten Sportvereinen zur Verfügung stehen und nicht der Quartierjugend zum außerschulischen Fußballspielen und als Treffpunkt.

Es reproduziert sich hier bei der Betrachtung der funktionalen Komponenten der Architektur die Sinnstruktur, die bereits bei den Basketballkörben zum Vorschein kam. Die Architektur des Schulhauses Hinter Gärten entfaltet keine Gestik, die auf die Eröffnung eines Möglichkeitsraums für autonome Tätigkeiten gerichtet ist. Im Gegenteil: Sie unterbindet diese. 


\subsubsection{Raumorganisation und Raumanordnung}

Beim Betreten des Eingangsbereichs zeigt sich, dass das Farbkonzept nicht nur eine Repräsentationsfunktion des Gebäudes darstellt. Die Farbigkeit des Gebäudes ist auch Teil der Binnenfunktion des Gebäudes. Dabei wirken Magenta und Rostrot der Fassadengestaltung zu den grellen Farbtönen des Innenbereichs vergleichsweise milde.

Im Folgenden wird in stark abgekürzter Form die Raumanordnung und insbesondere die Expressivität der Innenräume paraphrasiert. Zur Illustration und zur Abkürzung wurden Bilder eingefügt, die den Nachvollzug unterstützen.

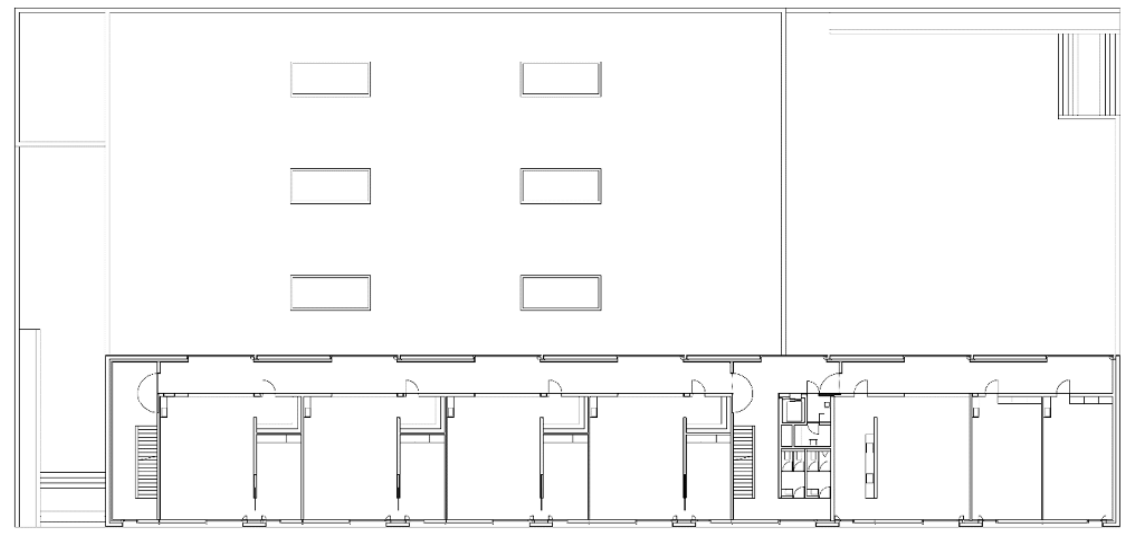

Abb. 20: Hinter Gärten. (C) Daniel Marques, Marques Architekten AG, Luzern

Das Schulhaus Hinter Gärten ist eine Korridorschule mit einbündigem Grundriss. Der Korridor ist an der Nordseite angebracht. Zwei Eingänge führen zu jeweils einem Treppenhaus. Die Räumlichkeiten sind in sieben gleich große Raumvolumen aufgeteilt. Als farbliche Raumfolge führt der Weg in die Klassenzimmer von den warmen roten Fassadentönen außen zum kühlen Blau der Pausenhallen. Die Räume, Wände, Decken, Boden sowie permanente und mobile Gegenstände sind in denselben Farben gehalten. Der Putz hat eine struktur- und konturlose Beschaffenheit, was den Effekt noch verstärkt. 
Die Räume sind in verschiedenen Farben gestrichen. Die Eingangsbereiche im Hausinnern und die vertikalen Erschließungen sind grün gestrichen, die horizontalen Erschließungen wiederum blau. Sämtliche Schulräume und die Turnhallen sind in einem grellen Gelb bemalt. Ausnahmen sind die Toiletten und Garderoben: Diese sind jeweils in unterschiedlichen, aber je Raum einheitlichen Farben gehalten. ${ }^{46}$

Die Raumeinheiten sind nach zwei Prinzipien verteilt. Zwischen den vertikalen Erschließungen sind vier gleichartige Klassenräume untergebracht. Jedes Klassenzimmer verfügt beim Eingang über eine halb offene Garderobe. Daran schließt der Hauptraum an, in den die Architekten Mobiliar für 24 Schüler_innen eingezeichnet haben. Davon abgetrennt ist etwa ein Viertel des Raumvolumens, in welchem vier zusammengeschobene Pulte ersichtlich sind.

Sämtliche Klassenzimmer sind in den Plänen in traditioneller Frontalbestuhlung angedacht. Auch die kleinen Gruppenarbeitsräume sind alle identisch gestaltet. Es zeigt sich hier, dass für die Architekten die Varianz von Unterricht relativ eng abgesteckt ist. Unterricht findet seriell, in konventionellem Frontalunterricht und in jedem Zimmer gleich statt. Die Wandtafeln sind fest eingebaut. Der Raum ist damit aus- und eingerichtet. Einzige Besonderheit im Vergleich zur Kernzelle eines traditionellen Klassenraums z. B. im Schulhaus Breitenrain sind die beiden zusätzlichen separaten Raumteile.

Das Raumkonzept wiederholt sich in einer Variation im östlichen Teil, der durch die Treppen von den Klassenräumen abgegrenzt ist. Die Variation besteht darin, dass die Aufteilungen der drei Bereich als je eigene Räume und damit fast doppelt so groß sind. Es sind ein großer Raum ohne Garderobe und zwei kleinere Räume, in denen jeweils eine halb so große Gruppe im selben Setting wie in den Klassenzimmern untergebracht werden kann.

Die Korridore sind eng, dasselbe gilt auch für die Treppenhäuser. Die Enge der Korridore und der Treppenhäuser erzwingt ein diszipliniertes Gehen in der Gruppe. Die mit viel Lärm und Bewegungen einhergehenden Fluchtbewegungen zu Unterrichtsbeginn und -schluss, wie sie in den großen Korridoren der Monumentalschulhäuser zu beobachten sind, sind hier kaum vorstellbar. Die festge- 
stellte asketische Schlichtheit und die Sparsamkeit des Außenraums bilden auch die Gestaltungsprinzipien des Innenraums.

Im Innenraum reproduzieren sich die dominanten Gestaltungsmerkmale des Außenbereichs, allerdings in potenziertem Maßstab. Die Farbigkeit der Räume ist in einer Farbsättigung und in grellen Farbtönen gehalten, welche die Fassade vergleichsweise matt erscheinen lassen. Auch das zweite Merkmal der Außengestaltung tritt verstärkt hervor: die polychrome Komposition durch monochrome Farben. Dabei sind die Räume integral in jeweils derselben Farbe gehalten, wodurch sie nicht nur in der Fläche monochrom sind. Der Effekt eines monochromen ,Gemäldes" potenziert sich als Farbwirkung in einem monochromen Raum. Der glatte, konturlose Putz hebt sich von Wänden, Böden und Decken die Farbwirkung hervor. Besonders irritierend ist, dass Möblierung, Stühle, Tische, Regale, Uhren, Lichtschalter, aber auch die Sportgeräte in der Turnhalle, etwa die Sprossenwand, exakt in derselben Farbe gehalten sind wie Wände, Diele und Böden. So verschwinden die Gegenstände quasi vor dem Hintergrund des Raums.

Da der Sinngehalt des Gebäudes so stark mit Farbe verwoben ist, wird eine nochmalige Beschäftigung mit Farben und deren Wahrnehmung unabdingbar (ausführlich siehe Kandel 2012; Kandel et al. 1996; Oevermann 2000b). Der Effekt der uniform-monochromen Farbgebung lässt die Konturen verwischen, da die visuelle Wahrnehmung eines Gegenstandes in Abhängigkeit zu den Merkmalen seines Hintergrundes steht. Farbe wird üblicherweise definiert als eine visuelle Empfindung, die aus dem Zusammenwirken äußerer (physikalischchemischer) und innerer (physiologisch und psychologischer) Gegebenheiten entsteht (Jahn \& Lieb 2008: 244). 


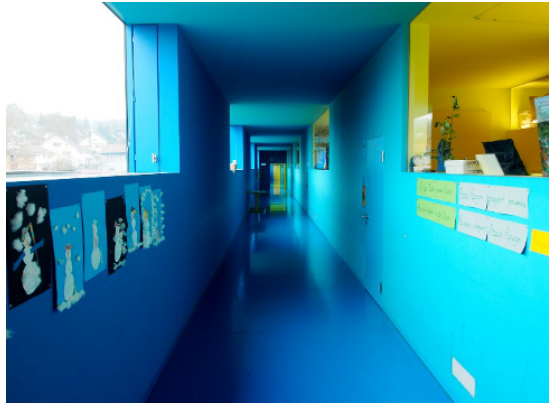

Abb. 21: Hinter Gärten. Korridor

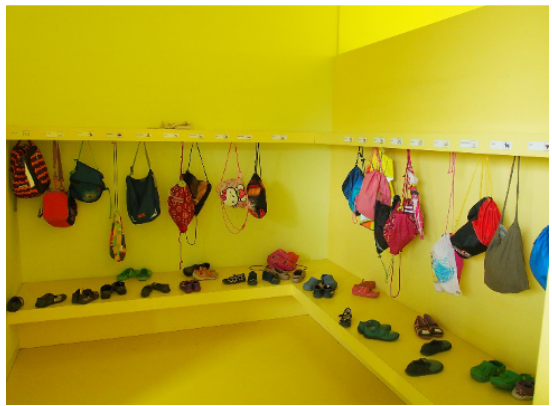

Abb. 23: Hinter Gärten. Garderobe

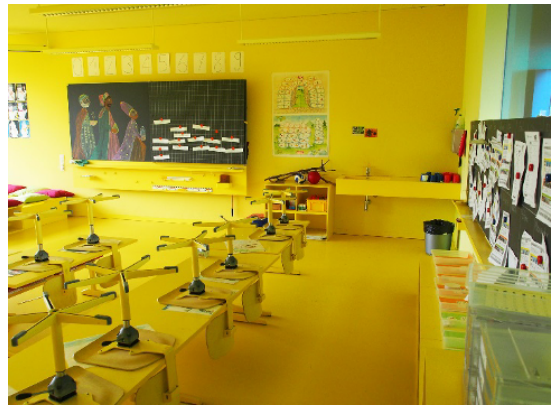

Abb. 25: Hinter Gärten. Klassenzimmer

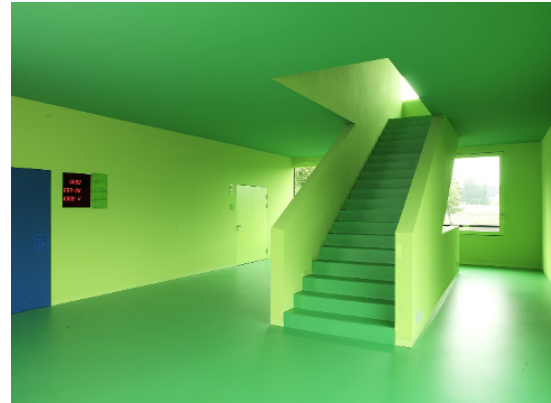

Abb. 22: Hinter Gärten. Detail. (C) H. Unger, Zürich

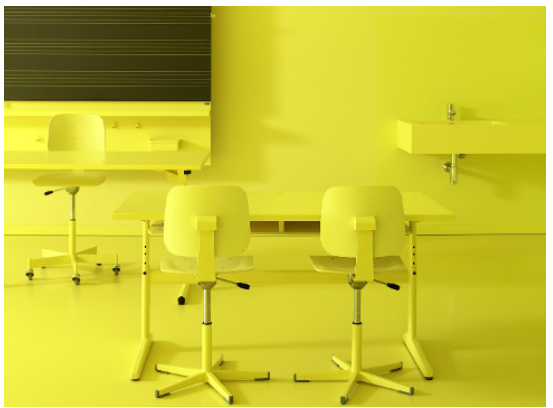

Abb. 24: Hinter Gärten. ㅇ Heinz Unger, Zürich

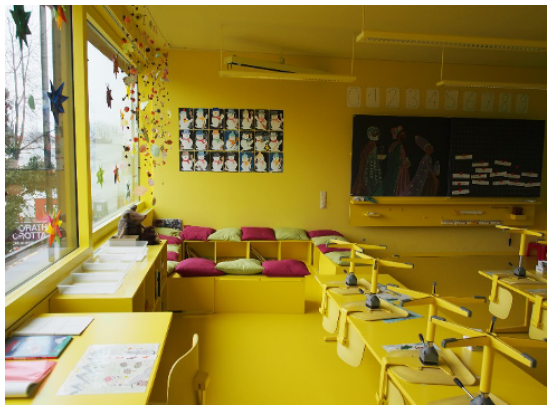

Abb. 26: Hinter Gärten. Klassenzimmer 
Für die Objektwahrnehmung sind Farbe(n) insbesondere in den Farbinformationen von Farbkontrast, Farbkonstanz ${ }^{47}$ und Farbantagonismus ${ }^{48}$ wesentlich. Die Farbwahrnehmung wird dabei definiert als der visuelle Eindruck, der es ermöglicht, zwischen zwei angrenzenden strukturlosen Flächen gleicher Helligkeit zu unterscheiden. Die Farbwahrnehmung erlaubt also, den Gegenstand in seiner Plastizität zu erfassen. Die Räume des Schulhauses Hinter Gärten sind so gestaltet, dass die räumliche Wahrnehmung und die Objektwahrnehmung im Prinzip verunmöglicht wird. Dies sieht man exemplarisch an der Fotografie, welche die Architekten als Architekturfotografie verwenden (Abb. 24). Der Raum und die konstant anwesenden Gegenstände werden durch die Farbe nahezu unsichtbar gemacht. Die Objekte verschmelzen durch die farbliche Täuschung mit ihrem Hintergrund, wie bei gut getarnten Tieren. Die Farbgebung manifestiert eine Sinnstruktur, die das ästhetische Empfinden der Farbe und die Farbqualität so in den Vordergrund rückt, dass der Raum als funktionale Umgebung und die Möbel als Artefakte mit ihren jeweiligen Intentionen, also auch die ikonologischen Embleme der Schule und des Unterrichts, also Pulte, Stühle, mit Ausnahme der Wandtafel, visuell verschwinden. Das Farbkonzept stellt einen Farbtotalismus dar.

Anzufügen ist, dass die monochrom-monotone Farbgebung auch den gegenteiligen Effekt hat. Denn sowie getarnte Gegenstände gleicher Farben optisch tendenziell weniger wahrgenommen werden, tritt alles, was in einem farblichen Kontrast dazu steht, schärfer hervor. Am Schulhaus Hinter Gärten ist dies dort ersichtlich, wo Kontraste durch die Architektur vorgesehen sind oder wo sie durch Aneignung, zum Beispiel in der Garderobe, geschehen. Die Anordnung der jeweils monochromen Räume verstärkt die Wirkung polychromer Farbkontraste dort, wo Öffnungen in den Wänden vorhanden sind, die Ein- oder Ausblicke in andere Räume ermöglichen. Die (fast) monochrome Fläche wird zu einem polychromen Gefüge. Andererseits gilt dasselbe für sämtliche Gegenstände und Personen, die sich im Raum aufhalten. Sowohl die Schüler_innen, die Lehrper-

47 Farbkonstanz meint, dass wir Objekte als solche erkennen, unabhängig davon, in welcher Farbe sie gehalten sind oder ob sie wechselnder Beleuchtung ausgesetzt sind.

48 Farbantagonismus bezieht sich auf die Anordnung von Gegenfarben, was eine stärkere Wahrnehmung zur Folge hat. 
son und sämtliche nicht dem Raum farblich zugehörigen Gegenstände werden durch den Kontrast besonders hervorgehoben. ${ }^{49}$

Die Rezeption von Farbe ist wesentlicher Teil der Architekturwahrnehmung. Für die Wahrnehmung von Bewegungen, für Tiefen- und Raumwahrnehmung, die relative Größenbestimmung und anderes mehr hat die Farbe eine zurückgestellte Bedeutung (Oevermann 2000b: 445). Wesentlich ist die Farbe für die genaue Bestimmung eines Objektes und für das ästhetische Empfinden. Dabei ist vor allem der Effekt der Farbkonstanz zentral. Für das Beispiel hier bedeutet das, dass das Schulhaus auch dann als Schulhaus erkannt wird, wenn es in unterschiedlichen Farben dargestellt wird.

Am Untersuchungsgegenstand zeigt sich jedoch das umgekehrte Phänomen. Auch wenn der Gebäudekomplex sich durch seine Farbigkeit von einem Industriebau abhebt, wirkt er von der Form her wie ein beliebiges Wirtschaftsgebäude. Damit reproduziert sich die Bedeutungsstruktur, die den ästhetischen Effekt des Gebäudes über die Funktionalität erhebt oder in der die Darstellung der Funktionalität des Gebäudes verweigert wird. Genauso wie das Gebäude sich von außen einer Erkennung und Prädizierung als Schule entgegenstellt, werden im Inneren die Gegenstände, die typischerweise für eine Schule stehen, mit einem optischen Trick, verwischt ${ }^{\text {. }}$

Die Kontraste sind dort gewollt, wo sie durch die Architektur, z. B. durch die transparenten Öffnungen, hergestellt werden. Oder umgekehrt gesagt: Die Funktion der Transparenz dient in erster Linie der Erzeugung von Farbkontrasten. Sie ist nicht für Ein- oder Ausblicke der Schüler_innen oder der Passanten gedacht. Das Gebäude repräsentiert sich selbst als Werk. Damit ist es Repräsentationsobjekt der Architekten, des Künstlers und der Bauherrschaft. Dies geht zulasten der Funktionalität für die Nutzer_innen.

Die Farbgestaltung wirft einige Fragen auf. Zu diskutieren ist erstens die Frage des Schulhauses Hinter Gärten als Kunstwerk: Inwiefern ist die Wirkung nicht nur im Überraschungseffekt krisenhaft, sondern dient der Initiierung eines Bildungsprozess? Zum Zweiten hat die Farbgestaltung unweigerlich einen routi-

49 Ob dieser Effekt physiologisch stärker ist, als dies in ,normalen` weißen Räumen der Fall ist, kann ich nicht beurteilen. Rein physiologisch gesehen müsste es zumindest dort so sein, wo Gegenstände in den Gegenfarben vorhanden sind. Für die Fragestellung dieser Arbeit sind die physiologischen Details jedoch nicht entscheidend. 
nehaften Charakter für die Nutzer_innen, welche die Architektur als Gebrauchswert nutzen und für die das Gebäude die Alltagsumgebung darstellt. Es stellt sich also in Bezug auf die Funktionalität des Gebäudes die Frage, ob und wie die Farbigkeit eine Lernhaltung oder Lernprozesse beeinflussen könnte, oder umgekehrt, welche Deutungen von Pädagogik und Sozialisation sich in dem Farbtotalismus ausdrückt.

Farbe ist laut Oevermann nur dann ein „Medium der Krisenkonstellation“ (2000b: 457), wenn eine der folgenden Bedingungen gegeben ist: Entweder es handelt sich um Gegenstände fremder Kulturen, die uns als Unbekanntes irritieren. Oder es handelt sich um ein gelungenes Kunstwerk. Das Kriterium für die Autonomie eines Kunstwerks ist der Umstand, ob der Betrachtende durch Überraschung und Irritation in den Zustand der Krise gebracht wird.

Bei monochromen Bildern ist dies in besonderem Maße gegeben, da eine bildliche Referenz per se entfällt. Daher zeichnen sich abstrakt monochrome und polychrome Bilder durch eine besondere Dekodierungsproblematik aus. Sie haben eine irritierende Wirkung, und die Wirkung kann nur perzeptiv erlebt werden. Monochrome Bilder verschließen sich quasi vollständig einer Versprachlichung. Das Gebäude ist unbestritten äußerst krisenevozierend. In dem Sinne ist es ein gelungenes Werk. Es ist kaum vorstellbar, dass man sich der sinnlichen Suggestion des Gebäudes verschließen kann. Dabei hat die Farbe einen doppelten Effekt. Einerseits im individuellen Erleben, aber auch in einem dadurch angestoßenen reflexiven Denkprozess. Der Farbtotalismus sowie die satten und grellen Farben sind dermaßen dominant und krisenhaft, dass sie zwangsläufig Fragen aufwerfen. Eine kleine Anekdote illustriert diese Auslegung: Eine Mitarbeiterin einer der am Bau beteiligten Firmen, mit der ich Kontakt hatte, wollte aus persönlichem Interesse unbedingt eine Einschätzung von mir, ob dieses Gebäude für Kinder denn überhaupt gesund sei. Sie war etwas besorgt, dass die Kinder in diesem Schulhaus eigentlich einem Experiment ausgesetzt seien, welches ethische Fragen aufwirft.

Diese Frage wird tatsächlich fast zwingend aufgeworfen. Sie kann hier nicht beantwortet werden. Es kann allenfalls überlegt werden, in welchen Kontexten satte monochrome Farben und ein Farbtotalismus erlebt werden. Vergleichbar wäre eine frisch verschneite Landschaft, das ewige Eis, die rabenschwarze Nacht, der Aufenthalt auf dem offenen Meer, in der Wüste oder in Monokulturen, wo man allenfalls in ähnlicher Weise einer eintönigen, dominanten Farbe 
ausgesetzt ist. Dies sind alles Kontexte, die besonders krisenhaftes Erleben evozieren. Es sind aber auch alles Kontexte, die für den Menschen lebensfeindliche Umgebungen darstellen.

Ein Schulhaus kann im Betrieb kein Kunstwerk sein. Es ist zwangsläufig Gebrauchsgegenstand. Die Räumlichkeiten sind abgesehen von ihrer Krisen evozierenden Wirkungen unter ihrer Funktionalität als ,pädagogische Architektur' zu betrachten. Die Dominanz der Ästhetik und die Absenz von leibbasierten Aneignungsmöglichkeiten kann als pädagogisches Konzept ausgelegt werden, in dem Bildungsprozesse primär im individualisierten, subjektiven und ästhetischen Erleben bestehen: in der Interaktion der Subjekte mit Architektur und Artefakten.

Es ist zu diskutieren, was das Farbkonzept bezüglich eines Unterrichtskonzeptes verkörpert. Wo liegen Gemeinsamkeiten und Unterschiede von Bildungsprozessen in Kunst und Schule? Gemeinsamkeiten zeigen sich bereits in den etymologischen Begrifflichkeiten. Der griechische Begriff scholè bedeutet Muße. Die ,Krise durch Muße' in der Kunstbetrachtung ist strukturaffin zu einem Bildungsprozess. Beides sind Prozesse, bei denen das Ziel der müßigen, zweckfreien Beschäftigung mit einem Gegenstand dazu führt, dass die gewohnten Erfahrungsroutinen in einen krisenhaften Zustand gelangen, mit dem Zweck, neue Erfahrungen zu machen und zu neuen Erkenntnissen zu kommen. Sowohl im pädagogisch angeleiteten Unterricht als auch in der Werksrezeption sind es äußere Stimuli, die eine Krise evozieren und gleichzeitig Lösungen zu deren Bearbeitung anbieten (ausführlich Oevermann 1996).

Die Kunst des Unterrichtens ist es, in einer pädagogischen Interaktion oder in einem arrangierten Lehr-Lern-Setting eine Frage so aufzuwerfen, dass die Schüler_innen selbsttätig oder mithilfe der Begleitung durch die Lehrperson zu Antworten gelangen und/oder praktische Problemlagen bearbeiten und lösen können. Die Aufgabe der Lehrperson ist also eine stellvertretende Krisenbearbeitung, indem sie das zu vermittelnde Wissen und Fertigkeiten in einer Art und Weise didaktisiert und transformiert, dass sie von den Schüler_innen idealerweise neugierig aufgenommen und selbsttätig oder mithilfe der Lehrperson bearbeitet werden können.

Analog dazu gerinnt im Kunstwerk eine subjektive Erfahrung des Künstlers, die in der Interaktion mit dem Kunstwerk suggestiv nacherlebt wird. Der Unterschied ist aber insbesondere darin zu sehen, dass das Kunstwerk eine zu Natur sublimierte Kultur, eine erstarrte Lebendigkeit, darstellt. Als Kunstwerk und 
ästhetische Komponente sind ästhetische Bildungsprozess der Kunsterfahrung durch Artefakte ausgelöst. Es ist zudem ein Prozess, der in der Interaktion mit dem Kunstwerk, aber in der Subjektivität des Empfindens vonstattengeht.

Demgegenüber ist die pädagogische Praxis eine interaktive Praxis, die in eine personale Interaktion eingebunden ist. Auch die selbsttätige Bearbeitung in der Lösung eines Aufgabenblattes oder von Hausaufgaben sind zwingend in eine Interaktion mit der Lehrperson eingebunden. Schulische Lern- und Bildungsprozesse sind Teil einer lebendigen Praxis, die situativ immer wieder aufs Neue hergestellt wird. Daher ist Pädagogik auch hochgradig kontingent.

Hier liegt das Unbehagen begründet, welches das Schulhaus Hinter Gärten tendenziell auslöst. Der Farbtotalismus entfaltet seine suggestive Wirkung umso stärker, je mehr das Gebäude für sich steht. Die sinnliche Suggestion vergrößert sich in der Absenz von allem, was nicht zum Farbtotalismus gehört. Damit steht die Ästhetik des Gebäudes konträr zur Funktionalität des Schulhauses. Jedes ins Haus getragene Objekt, jede Aneignung und sogar jedes auch nur temporär herumstehende Subjekt wirkt gegenüber der überdominanten Farbwirkung frevelhaft und störend.

Durch das Verwischen der Raumkontur werden vermutlich auch die Positionalitäten der Personen visuell weniger stark zum Ausdruck kommen. Die Handlungsdispositionen, die durch die Möblierungen präkonfigurierend vorhanden sind, bleiben bestehen. Sie werden lediglich mittels des Farbschleiers verdeckt. Damit entfaltet die Farbe eine Tarnfunktion. Durch die Farbigkeit der Räume wird optisch zum Verschwinden gebracht, was faktisch vorhanden ist: die Schule in ihrem räumlich-physischen Setting einer ganz spezifischen Form.

Es ist anzunehmen, dass sich für die Schüler_innen und das Personal relativ bald eine Normalität der Farbwahrnehmung einstellt und die Farbigkeit des Raums in den Hintergrund rückt sowie generell die Umgebung als dauerhafter Hintergrundstrom der Eindrücke in der Regel außerhalb des bewussten Aufmerksamkeitsfokus liegt (vgl. Steets 2015: 71 ff.). Dabei haben Farben eine Wirkung auf unser Gefühlsleben (Kandel 2012: 286 f.). Farben lösen emotionale Effekte aus und können Stimmungen beeinflussen: Dunkle Farben wirken düster, helle Farben wirken heiter. So gibt es verschiedene Untersuchungen zu Farbeffekten auch in pädagogischen Räumen (Rodeck et al. 2002: 84) und Empfehlungen, diese zum Beispiel in warmen Farbtönen zu halten, um eine Atmosphäre von Wohlbefinden und Geborgenheit zu schaffen. Im Alltagsgebrauch wird die 
Farbwahrnehmung allenfalls als sublime und unbewusste Einflussgröße einen Effekt entfalten. Die Raumorganisation und die Möblierungen werden als Präkonfiguration von Beziehungen und Praktiken eine weitaus höhere Effektivität auf das Handeln entfalten.

Das Raumprogramm des Schulhaus Hinter Gärten verfügt über verschiedene Raumvolumen in unterschiedlichen Raumgefügen (Abb. 20). Erkennbar sind die Klassenzimmer ins Zentrum gerückt. Sie werden von den Erschließungen gerahmt. Die Fachräume, Büros und die Sanitärräume befinden sich räumlich davon abgetrennt. Sie bilden damit fast einen eigenen, abgegrenzten Trakt. Eine Unterteilung nach Funktionsräumen findet sich auch in der vertikalen Gliederung: Im Untergeschoss befinden sich die Turnhalle und die Garderoben, also die Räume für leibliche und körperliche Betätigungen. Sie bilden metaphorisch gesprochen das Fundament. Und wie das Fundament, sind sie von außen gesehen (fast) unsichtbar. Im Erdgeschoss sind Aula, Tagesstruktur, Bibliothek und Musikraum untergebracht. In den oberen Geschossen sind die einheitlichen Klassenräume untergebracht.

Mit Ausnahme der Toiletten und den Garderoben sind die Räume gelb. Die vertikalen Erschließungen sind grün und die horizontalen Erschließungen blau gehalten. Auf eine detaillierte Analyse des Raumprogramms wird aus Platzgründen verzichtet. Grundsätzlich kann festgehalten werden, dass die Grundstruktur der Raumorganisation Ähnlichkeiten zum Schulhaus Breitenrain aufweist. Zumindest sind keine grundlegenden Unterschiede im Raumprogramm zu finden, wie dies das radikale Farbkonzept und der Umstand, dass die Schule nicht als Schule erkennbar ist, suggerieren. So findet sich kein größerer Anteil an Räumen für ästhetische oder musische Betätigung, was zu erwarten wäre. Aber auch in pädagogischer Hinsicht sind keine Anhaltspunkte für grundsätzlich differente Unterrichtskonzepte zu finden, die die Radikalität der Architektur spiegeln würden. Es gibt weder Anzeichen für ,offene' Lernformen noch für Konzepte von Lern- und Bildungslandschaften oder Lernateliers, wie sie im aktuellen pädagogischen Diskurs für eine ,gute Schule gefordert werden. Die maßgeblichen räumlich konstituierten Differenzen zum Raumgefüge des Schulhauses Breitenrain sind in den Raumprogrammen, also der Binnengliederung der Klassenzimmer zu sehen. Im Folgenden wird darauf fokussiert. 


\subsubsection{Der Klassenraum als Schema mit Variationen}

Exemplarisch wird eines der Klassenzimmer betrachtet (Abb. 20-26). Der Klassenraum ist ein Raumgefüge von Garderobe, Hauptraum und Appendix in Form eines Gruppenraums. Beim Eingang betritt man einen abgetrennten Teil, die Garderobe. In älteren Schulen - aber auch in vielen öffentlichen Gebäuden wie z. B. im Theater - sind die Garderoben in den öffentlichen Erschließungsräumen platziert. Die Kleidung wird im öffentlichen Außenbereich abgelegt, bevor die inneren Räume in der dem Anlass angepassten Aufmachung betreten wird. Die Garderobe ist im Schulhaus Hinter Gärten in die innere Privatheit des Klassenverbundes verlegt, wie dies in der Regel auch in Wohngebäuden der Fall ist. Damit ist die Trennung von Öffentlichem und Privatem viel abrupter, was eine größere Abschottung und eine intimere Sphäre innerhalb der Klasse suggeriert. Trotzdem gibt es eine binnenräumliche physische Trennung des Garderobenbereiches von dem Klassenzimmer. Die Garderobe ist halb offen gestaltet und so platziert, dass sie ausschließlich von dem Bereich einsehbar ist, der für die Lehrperson vorgesehen ist. Der Blick von der Tür auf den Teil der frontal angebrachten Wandtafel ist gegeben und vice versa.

Der eigentliche Unterrichtsraum ist in einen großen Hauptraum und einen kleinen Nebenraum unterteilt. Der Nebenraum ist mir einer breiten Öffnung verbunden, die bei Bedarf mit einer Schiebetür abgeschlossen werden kann. Damit kann er funktional different genutzt werden, als Teil des Klassenzimmers oder als geschlossener Raum.

Das eigentliche Klassenzimmer wird durch die mobile und immobile Möblierung in fünf Bereiche unterteilt: die Aufenthaltsfläche der Lehrperson ,vorne", die durch die Wandtafel und Instruktionsgegenstände markiert ist. Als komplementierendes Gegenstück dazu ist der Großteil der Fläche mit hufeisenförmig angelegten Pulten der Schüler_innen bestückt. Davon abgesetzt finden sich fest installierte, quadratisch angeordnete Sitzbänke. Zudem ist eine zweite Anschauungsfläche auf der Seite festzuhalten, mit Ablagen und Stauraum kombiniert. Als Letztes findet sich ein einzelnes Pult, das sich durch die Blickrichtung aus dem Fenster von den anderen Pulten abhebt. Die Bereiche sind jeweils mit eigenen Funktionen verbunden, die insgesamt als ein ,Unterrichtssystem“ zu begreifen sind. Sie stehen jeweils für unterschiedliche Interaktionsformen und -ordnungen und Formen der Aneignungen. 
Die Ausrichtung des Klassenraums ist fix. Die fest installierte Tafel konstituiert deutlich ein ,Vorne‘. Die Raumkonfiguration stellt ein Setting mit einer eindeutigen Interaktionsordnung dar. Analog zu einer Bühne steht die Lehrperson vorne im Zentrum, und die Schüler_innen folgen in der Rolle des Publikums der Performanz der Lehrperson. Die schulische Unterrichtung findet als Interaktion statt: Vorne wird etwas vorgeführt oder vorgezeigt. Das Mittel der unterschiedlichen Gestaltungsmöglichkeiten, was vorne passiert, stellt die Wandtafel dar. Sie ist das zentrale Instrument schulischer Stoffvermittlung, metaphorisch gesagt das Bühnenbild, vor welchem sich die Lehrperson in unterschiedlichen Rollen darstellt und an der sie verschiedene Inhalte anbringt und entwickelt. Die Funktion der Tafel ist nicht ,nur " die Möglichkeit, wechselnde Bildungsinhalte geschrieben und dargestellt auf ihr zu entwickeln. Die Tafel eignet sich auch zur analytischen ,Zerlegung ' und der Illustrierung komplexer und zum Zwecke der Didaktik konstruierter Bildungsinhalte. In Bezug auf die Interaktionsordnung sind die Rollen einer Meister-Novize-Beziehung verteilt. Die Grundoperation pädagogischer Tätigkeit ist das ,Zeigen', das ,Vorzeigen' oder ,Vorführen'. Die Schüler_innen nehmen entweder eine passive Rolle als Publikum oder eine nachahmende Rolle ein. In beiden Fällen finden die Lern- und Bildungsprozesse als extrinsisch initiierte Interaktionen statt, in denen Wissensinhalte über das Zuhören und Zusehen und über die übende Nachahmung internalisiert werden. Dieser Sachverhalt gilt auch unter dem Umstand, dass die Anschauungsfläche und Lehrpersonen nicht ausschließlich von der Lehrperson ,bespielt ${ }^{\star}$ werden muss, sondern auch Schüler_innen auf die Bühne gerufen werden. Der Umstand, dass Wandtafel und Kreide als Besonderheit immer wieder von Neuem genutzt werden können, birgt auch die Möglichkeiten des Ausprobierens und des Korrigierens. Dies macht die Anschauungsflächen zum bei Schüler_innen oft gefürchteten Ort, wo man vor Publikum auf einer Bühne stehend sich bewähren muss oder auch vorgeführt wird. Festgehalten soll werden, dass die Raum(an)ordnung eine Bühnenanordnung darstellt, in welcher die Interaktionen eindimensional gerichtet sind.

Die Anordnung der Schüler_innen ist eine hufeisenförmige Sitzordnung. Dies stellt eine Transformation der rein seriellen Ordnung dar, wie sie durch die Subsellien im 19. Jahrhundert festgelegt wurden. Die Blickrichtungen hin zum Anschauungsraum bleiben erhalten. Die Klasse ist jedoch so angeordnet, dass die Schüler_innen nicht mehr als individualisierte Schüler vereinzelt auf ihren 
,Rängen“ sitzen. Die Peers und damit die Klasse werden zu einem zentralen Bezugspunkt der Raumordnung.

Dieses Prinzip findet sich auch beim zweiten Bereich, wo sich vier fest installierte Bänke mit Sitzkissen befinden. Die Bänke sind in zweifacher Hinsicht bemerkenswert. Zum Ersten ist mit dem vergleichenden Blick auf die Pläne der Architekten feststellbar, dass dieser Ort für das Lehrerpult gedacht ist. Die subjektive Intention der Architekten war die Raumkonfiguration eines konservativtraditionellen, lehrerzentrierten Unterrichts. Dazu ist festzuhalten, dass das Lehrerpult, sofern es überhaupt vorhanden ist, faktisch gänzlich anders platziert ist.

Die vier quadratisch angeordneten Bänke stellen eine Anordnung dar, die in der Pädagogik Kreis genannt wird. Der Kreis ist seit Fröbels Zeiten eine feste Institution des Kindergartens. In den letzten Jahren ist dieses räumlich in die Schulen diffundiert. Der Kreis impliziert und symbolisiert eine Interaktionsform in einer Gruppe, wobei explizit die gesamte Gruppe adressiert wird. Es wird eine Einheit nicht nur symbolisiert, sondern sie soll räumlich erlebt werden. Dabei werden dem Kreis gemeinschaftsstiftende und demokratiefördernden Wirkungen zugesprochen (für eine ausführliche Diskussion siehe Kuhn \& Magyar-Haas 2011). Er soll für die einzelnen Teilnehmende öffnend wirken und damit die Integration fördern. Oft ist der Kreis als Ritual des Ankommens und der Verabschiedung die bevorzugte Sozialform. Aber auch als Ort angeleiteter Spiele, in denen motorische Komponenten zentral sind, gemeinschaftlicher Tätigkeiten wie des Singens, des gemeinsamen Rezitierens u. a. m. In der Schule wird der Kreis hingegen öfter als Besprechungsform angewandt. In den normativen Programmatiken der Literatur, die sich mit Kreissequenzen befassen, werden vor allem Aspekte hervorgehoben, die die soziale Kohäsion der Klasse fördern.

Der konkrete ,Kreis‘ im Bild ist mehrfach irritierend. Im Sitzkreis gibt es lediglich 16 Plätze, was für die Klasse und für die Konzeption des Klassenzimmers zu klein ist. Denn die 21 Bilder der Schneemänner an der Wand lassen auf mindestens 21 Schüler_innen schließen. Der Kreis ist nur für einen Teil der Klasse konzipiert, um als Interaktionsstruktur für längere Unterrichtseinheiten sinnvoll zu sein. Es ist damit eine gewisse Konterkarierung der pädagogischen Intention festzustellen, wenn der Ort der Klassengemeinschaft nicht alle Mitglieder der Klasse aufnehmen kann. Diese Konterkarierung findet sich auch symbolisch. Einerseits indem der Kreis unvollständig ist: Es fehlen zwei Elemente, die 
zugunsten einer bequemeren Erreichbarkeit weggelassen werden. Andererseits realisiert sich die Quadratur des Kreises in der Realisation des Kreises als Quadrat.

Der dritte räumliche Bereich mit einer eigenen Funktionalität ist die nordseitige Wand. An ihr ist eine weitere Wandtafel angebracht, die im Rücken eines Teils der Schüler_innen liegt. Unter der Wandtafel sind fest installierte Ablagen, auf der Arbeitsmaterialien in Form von Blättern angeordnet sind. Die Wandtafel wird genutzt, um Blätter aufzuhängen. Die Ablage und die Wandtafel intendieren, dass sich die Schüler_innen selbst mit Arbeitsblättern bedienen und selbst die fertigen Produkte aufhängen.

Dieser Bereich realisiert also eine Interaktionsordnung geradezu konträr zu derjenigen der hufeisenförmigen Pulte, in der jede Schülerin, jeder Schüler an einem Arbeitsblatt in der Regel für sich alleine arbeitet. Dies bedeutet nicht, dass dieser Bereich nicht auch anders genutzt werden kann und wird. In diesem Sinne stellt die Architektur Möglichkeiten her, die den Spielraum der Lehrpersonen erweitern. Eine gelungene Architektur soll Möglichkeiten insofern eröffnen, als die Klassenzimmer nach den Bedürfnissen und Unterrichtskonzepten der Lehrpersonen eingerichtet werden können. Denn unterschiedliche Unterrichtskonzepte verlangen nach anderen räumlichen Settings. In dem exemplarisch betrachteten Klassenzimmer findet sich eine Struktur, die zur Struktur und Unterrichtkonfiguration auf den Planskizzen differiert.

Wesentlich scheint, dass in diesem Klassenraum die pädagogische Interaktion sich über ganz spezifische Artefakte - Arbeitsblätter - vermittelt vollzieht. Dies hat eine doppelte Implikation. Zum einen die, dass Unterricht über Dinge vermittelt wird. Unterricht vollzieht sich nicht zwingend mündlich, unter physischer Anwesenheit und in einer pädagogischen Interaktion. Diese ist zwar weiterhin nicht ausgeschlossen. Der Unterricht hat hier aber eine Form, in der Wissen primär über Artefakte angeeignet wird. Zum Zweiten haben diese Dinge eine ganz spezifische Form: Es sind Arbeitsblätter. Damit liegen diese außerhalb der Architekturanalyse, sie werden auch nicht als solche analysiert. Sie können in ihrer Form aber in Analogie zum Kunstwerk als erstarrte Lebendigkeit betrachtet werden. Doch wird vorerst der erste Fall fokussiert.

Die Architektur lässt schließen, dass das Programm des Raums eine Unterrichtsform vorsieht, in der die Schüler_innen Aufgabenblätter holen und diese in der hufeisenförmigen Sitzordnung jeweils alleine oder allenfalls mit den Nach- 
barn bearbeiten. Der Umstand, dass Blätter selbstständig geholt werden, impliziert zwingend, dass eine gewisse Differenz in der Bearbeitung vorhanden ist. Entweder die Schüler_innen wählen ein Blatt unter verschiedenen, oder die Differenz besteht in dem unterschiedlichen Tempo, in dem die Aufgaben bearbeitet werden. Die Grundform liegt in beiden Fällen in der Arbeitsweise, dass die Schüler_innen jede/r für sich die Handlungsanweisungen lösen, die durch die Arbeitsblätter gegeben sind. Der Lern- und Bildungsprozess findet statt, indem die Schüler_innen sich das Wissen oder die Kompetenzen über die Beschäftigung mit einer ,erstarrten Lebendigkeit" selbst aneignen. Die Lehrpersonen sind situativ dann vorhanden, wenn Schüler_innen Hilfe benötigen. Die Intention des pädagogischen Konzepts ist, dass die Schüler_innen sich intrinsisch motiviert und selbsttätig aus ihren Interessen herausbilden sollen. Dabei ist festzuhalten, dass das Wissen oder die Wissens- und Vermittlungsformen in einer ganz spezifischen, pädagogisch vorkonfigurierten Form vonstattengeht: So wie sich das im Programm des Raums in Form einer fest installierten Theke für Arbeitsblätter manifestiert. Arbeitsblätter stellen Lehrmittel dar, die im Gegensatz zum althergebrachten Lehrbuch lose sind. Sie repräsentieren damit stärker eine selbsttätige Bearbeitung derselben. Dies impliziert, dass die Arbeitsblätter in einer spezifischen Weise konstruiert sind. Arbeitsblätter müssen aus ihrer Logik heraus eindeutig sein: Es sind eindeutige Handlungsanweisungen, die von ihrer Struktur her eine pädagogisch relevante Frage aufwerfen und gleichzeitig vorgezeichnete, schrittweise Wege zu deren eindeutiger Beantwortung vorzeichnen. Es sind Routinen, und sie sind in der Regel auf die Einübung routinehafter Lernprozesse gerichtet. Zumindest ist es außerordentlich schwer, ein Arbeitsblatt zu entwerfen, das einen krisenhaften und damit kontingenten Bildungsprozess evoziert (zur Kontingenz von Unterricht siehe Combe 2015). Dieser bedarf aber zwingend einer nachträglichen pädagogischen Interaktion aus Gründen der Geltungsüberprüfung. Der große Teil der mir bekannten Arbeitsblätter sind im besten Falle eindeutige, rational stringente, auf standardisierte Lösungswege gerichtete Aufgaben. ${ }^{50}$ Einerseits regen diese explizit zu einer kognitiven Leistung an, und

50 Im schlechteren Fall, was relativ oft der Fall ist, sind sie uneindeutig oder unklar formuliert, und inhaltlich oft auch schlichtweg falsch. Gelungene Arbeitsblätter sind jedenfalls äußerst anspruchsvoll in der Erstellung. 
andererseits wird mit diesen - so die These - implizit eine Gefolgschaft gegenüber durch Artefakte erzeugte Handlungsdispositionen einsozialisiert. Die Uniformität - die sich zum Beispiel in Form uniformer Praktiken in der Herstellung der fast identischen Schneemänner an der Wand zeigt - wird u. a. über Handlungsanweisungen durch Artefakte hergestellt. Von den Subjekten verlangt dies einen wesentlich selbstdisziplinierteren Umgang, als dies bei einem lehrpersonenzentrierten Unterricht der Fall ist, wie er am Schulhaus Breitenrain rekonstruiert wurde. ${ }^{51}$

Diese Form von Unterricht konstituiert auch die pädagogische Interaktion zwischen Lehrperson(en) und Schüler bzw. Schülerin beziehungsweise zwischen Lehrperson und Klasse neu. Es ist zu vermuten, dass die Interaktion weniger auf den Inhalt des Arbeitsblattes gerichtet ist als mehr auf die Prozesse, wie die Aufgaben gelöst werden: Anstelle eines Wissens bzw. der Wissensvermittlung treten (Bearbeitungs-)Kompetenzen. Die Lehrperson steht den Schüler_innen unterstützend zur Seite, wenn sie Hilfe brauchen. Es ist im Vergleich zum lehrerzentrierten Unterricht eine Verlagerung festzustellen, indem die Vermittlung nicht uniform über die gleichzeitige kollektive Adressierung der Klasse verläuft. Die uniformen Inhalte und Wissensbestände werden über uniforme Artefakte vermittelt. Die Absenz des zentral arrangierten Lehrerpultes verweist darauf, dass die Position der Lehrperson oder der Lehrpersonen im ganzen Raum dort ist, wo sie gebraucht wird.

Dieser Umstand manifestiert sich beim vierten räumlichen Bereich und ist zugleich zu hinterfragen. Der vierte Bereich entlang des Fensters hat eine Einrichtungsstruktur, die mit der Theke entlang des Eingangsbereichs korrespondiert. Auch hier gibt es eine Theke, auf welcher vier leere Behälter ersichtlich sind. Es scheint also der Ort für die Abgabe der Blätter zu sein.

Zudem befindet sich hier ein einzelnes Pult, das mit Sicht aus dem Fenster platziert ist. Die Anordnung des Pultes könnte in einer Lesart darauf hinweisen, dass es eine Schülerin oder einen Schüler gibt, der/die außerhalb des Klassenver-

51 Dabei werden keinerlei Schlüsse auf den faktischen Unterricht gezogen. Dies ist mir wichtig festzuhalten. Auch im Schulhaus Breitenrain findet mutmaßlich auch ein sogenannter individualisierter Unterricht über Arbeitsblätter statt. Der Unterschied ist eher darin zu sehen, dass den Räumlichkeiten des Schulhauses Hinter Gärten dies als Programm stärker eingeschrieben ist. 
bundes situativ separiert wird. Dagegen spricht, dass das Pult etwas größer ist als die übrigen Pulte. Es kann sich daher auch um das Lehrerpult handeln. Die Platzierung mit Sichtrichtung aus dem Fenster unterstreicht ziemlich drastisch die oben explizierte Sinnstruktur. Unter dieser Annahme ist die Lehrperson zwar physisch anwesend, doch weder ist sie als Bezugspunkt der Klasse platziert, noch hat die Lehrperson eine Kontrolle darüber, was die Klasse macht. Dies würde auch implizieren, dass die Schüler_innen aktiv die Lehrperson aufsuchen müssen. Es lässt sich nicht zweifelsfrei entscheiden, welche der beiden Deutungen zutrifft. Eine dritte - nicht sehr sparsame - Lesart ist, dass die Lehrperson ihr Pult vor allem außerhalb der Unterrichtszeiten nutzt und sich dafür einen Fensterplatz aussucht.

Der Abgabeort und der Umstand, dass Abgabe und Holen räumlich getrennt sind, deuten eher auf das Lehrerpult hin. Als räumliches Arrangement ist dieses Zimmer so eingerichtet, dass der Unterricht mindestens zu gewissen Teilen als individualisierte, selbsttätige Bearbeitung eines Gegenstandes stattfindet, der über Artefakte in Form von verschrifteten Aufgaben erfolgt. Die pädagogische Interaktion findet demgemäß situativ dort statt, wo die Arbeitsblätter nicht selbstständig gelöst werden. Es sollen hier keine Spekulationen zu den Praktiken der Lehrpersonen in diesem Schulhaus angestellt werden. Was anhand der Bilder aber festgestellt werden kann, ist einerseits, dass eine Raumanordnung vorhanden ist, die einen arbeitsblätterbasierten, sogenannten individualisierten Unterricht impliziert, und andererseits, dass im Raum keine Gegenstände vorhanden sind, die zu einer sinnlich sensomotorischen Erschließung von Wissensinhalten anregen. Die einzigen Gegenstände, die zu haptisch-sinnlicher Erfahrungen anregen, sind ein Stofftier, zwei Bälle und zwei Äste. Im Großen und Ganzen ist der Raum ein ziemlich leerer ,Behälter`.

Es bleibt ein letztes Raumsegment zu betrachten. Eine weitere Räumlichkeit und ein gesonderter Bereich ist der Appendix, der Gruppenarbeitsraum. Auch hier ist eine Differenz zwischen Intention durch die Architektur und der tatsächlichen Nutzung festzustellen. Gemäß den Plänen ist der Raum für die Hälfte der Klasse gedacht. Beim exemplarisch erhobenen Klassenzimmer ist der Raum für ungleich weniger, nämlich maximal vier Schüler_innen eingerichtet. Der Raum macht einen kargen Eindruck und scheint wenig genutzt. Im Unterschied zum großen Klassenzimmer sind auch keine selbst gemachten Erzeugnisse an den 
Wänden zu finden. Der Raum ist mit einer Wandbreite eingebauter Schränke explizit als Lagerraum gedacht.

Zusammenfassend kann über das Raumprogramm und das Programm des Raums gesagt werden, dass das Klassenzimmer verschiedene, funktional unterschiedliche Bereiche herstellt, in denen sich verschiedene Tätigkeiten in verschiedenen Interaktionsformen und -ordnungen realisieren. Die Architektur eröffnet eine weitaus größere Varianz an Möglichkeiten, als dies in der Enge des Klassenzimmers im Schulhaus Breitenrain der Fall ist. Dabei ist anzufügen, dass sich viele Funktionsbereiche (Kreis, Materialregal usw.) auch im Schulhaus Breitenrain finden. Sie sind dort aber offensichtlich nachträglich, in mobiler Weise und/oder unter äußerst engen Verhältnissen errichtet worden.

Die unterschiedlichsten Bereiche, die verschiedenen Interaktionsformen und -ordnungen sind räumlich darauf ausgerichtet, dass mehr als eine Lehrperson anwesend ist oder sein kann. In diesem Sinne ist das Abbild dieses Raumprogrammes auch ein Abbild einer Klasse, wo die Vermittlung nicht uniform über den traditionellen, lehrpersonenzentrierten Unterricht geschieht. Der objektive Sinn, wie er sich in den Plänen der Architekten zeigt, verweist auf ein uniformes Programm traditionellen Unterrichts. Das zufällig ausgewählte Klassenzimmer hingegen zeigt exemplarisch eine weitgehend individuierte Aneignung durch die Lehrpersonen, die den Raum gemäß ihren pädagogischen Anforderungen eingerichtet haben.

\subsection{Fazit - Repräsentation krisenhafter Muße und Funktionalität einer Lernfabrik}

Im Zentrum der rekonstruierten Sinnstruktur des Gebäudes steht die Intention als Kunstwerk. Als krisenhaftes Werk betrachtet stellt das Gebäude ein gelungenes Werk dar. Es ist selbstreferenziell und löst in seiner erstarrten Lebendigkeit eine starke Suggestion aus. Dies gilt noch mehr für den Innenbereich des Gebäudes. In der Betrachtung der Gebäude als Repräsentation von Bauherrschaft und der Funktionalität kann konstatiert werden, dass sich die Bauherrschaft - die Gemeinde Riehen - als Mäzenin repräsentiert, die der Öffentlichkeit ein Werk zur Verfügung stellt. Diese starke Suggestivität als künstlerisches Gebilde überlagert die eigentliche Funktion des Gebäudes als Schule. Das Gebäude ist von außen 
nicht als Schulhaus erkennbar. Der Komplex hat die Form eines Wirtschaftsgebäudes, welches sich einen Repräsentationsanstrich gibt, der sich mit einer Affinität zu Kunst, Kreativität und Innovation verbindet. Damit nimmt das Gebäude Anleihe bei einer Hightech-Ökonomie, einem kunstnahen Dienstleistungsbetrieb oder einem Kulturbau.

Auch im Innenraum ist das Farbkonzept so gewählt, dass die Ästhetik zwangsläufig in den Vordergrund rückt. Eine Farbintensität ist überdominant und wird zum Farbtotalismus. Damit werden die sinnbildlichen und sinnbildenden Gegenstände der Schule übertüncht. Die ikonischen Symbole des Unterrichts werden visuell zum Verschwinden gebracht. Insgesamt überlagert die Atmosphäre der Architektur als Kunstobjekt die Funktionalität. Oder umgekehrt gesagt: Physisch-materiell stellt das Gebäude die Minimalanforderungen der Funktionalität in einer schlichten, schnörkellosen und zweckrationalen Weise her. Die Intention als Kunstwerk kommt überdominant zur Geltung.

Als Effekt resultiert daraus, dass sich das Gebäude einer Aneignung versperrt. Charakteristisch für das Gebäude ist, dass es nicht nur keine Handlungsintentionen durch Architektur herstellt, mit Ausnahme der Kunstbetrachtung. Im Gegenteil werden alle Handlungen, die auf eine Aneignung des Gebäudes zielen, unterbunden, mit Ausnahme derjenigen der Kunstbetrachtung. So sind nur wenige Elemente identifizierbar, die überhaupt Hinweise liefern, dass es ein Raum und ein Bau für Sozialisanden ist. Es sind - mit ganz wenigen Ausnahmen keine durch Architektur hergestellten Handlungsimpulse identifizierbar, die sich explizit an Kinder und Jugendliche zur selbsttätigen Aneignung richten. Es werden kaum Handlungsintentionen ermöglicht, die erfahrungs- oder körperbasiert sind und die die Autonomie von Kindern und Jugendlichen befördern. Dies betrifft nicht nur Spiel- und Sportgelegenheiten, sondern überhaupt einen Raum, in denen sich die Sozialisanden aufhalten können.

In der Interpretation der Architektur als pädagogisches Konzept wird erwartet, dass die Schule das ästhetische Empfinden ins Zentrum von Bildungsprozessen stellt. Bildungs- und Lernprozesse sind Erfahrungen, die primär sinnlich durch einen äußeren Stimulus angeregt werden. Das Schulhaus repräsentiert damit eine individualisierte sinnliche Aneignung, so wie die Architektur insgesamt primär einen kognitiv-perzeptiven Stimulus erzeugt. Als Schulhaus wird die Werksbetrachtung um ihrer selbst willen nicht Teil der pädagogischen Interaktion sein. Sie ist Hintergrundumgebung. Das Schulhaus drückt insofern ein päda- 
gogisches Konzept aus, das vor allem aus individueller Aneignung über ästhetisch aufbereitete Gegenstände besteht.

Diese Sinnstruktur wurde auch in der Rekonstruktion des Raumprogrammes und des Programms der kleinräumigen Grundzelle des Schulhauses rekonstruiert. Der Klassenraum eröffnet aus sich heraus eine Vielzahl von Interaktionsformen in unterschiedlichen Gruppen- und Interaktionszusammenhängen. In diesem Sinne eröffnet die Architektur zahlreiche Möglichkeiten der Unterrichtsgestaltung und theoretisch auch der Aneignungsformen durch die Schüler_innen. Es zeigt sich, dass einerseits die Kennzeichen eines traditionellen und konventionellen Unterrichts vorhanden sind: Räume im Klassenverbund der Kohorte, eine Möblierung, die nach den Prinzipien des seriellen Unterrichtens arrangiert ist, die klassischen ikonenhaften Mittel des Unterrichts wie die Wandtafel und anderes mehr. Andererseits wurde aus dem Raum zusätzlich ein Unterrichtskonzept rekonstruiert, in welchem die Schüler_innen sich relativ autonom und selbstreflexiv bilden, indem sie durch Artefakte angeleitet werden. Die pädagogische Interaktion findet situativ assistierend statt. Sie ist als Normalform in spezifische sozialräumliche Settings ausgelagert.

Zwei Merkmale sollen hier nochmals hervorgehoben werden. Aus dem räumlichen Setting wurde eine Unterrichtsmethodik rekonstruiert, die auf eine individuelle Aneignung von pädagogisch arrangierten Lernprozessen in Form von Handlungsanweisungen in Papierform ausgerichtet ist. Dies entspricht einer Doktrin, die sich in der Schule als zeitgemäße Unterrichtsmethode durchsetzt. An die Stelle eines Unterrichts, in welcher eine Kohorte in uniformen Tätigkeiten denselben Lerngegenstand behandelt, wird ein ,individualisiertes“ und „(selbst-)reflexives“ Lernen in selbst organisierten Lernumgebungen fordert (z. B. bei H. Meyer 2013; Watschinger \& Kühebacher 2007). Es ist ein Prinzip, das als (je nach Konzept ergänzender oder substituierender) Gegensatz zum Frontalunterricht in der Kohorte gedacht und konzipiert ist, um der Heterogenität bezüglich Wissen, Geschick, Fähigkeiten usw. innerhalb der Klasse gerecht zu werden. Ein individualisierter Unterricht verlangt nach Strukturen, die eine Binnendifferenzierung des Unterrichts auf die Individuen erlaubt.

Im Vergleich zum Schulhaus Breitenrain und den älteren Schulhäusern in Schmitten (Kap. 7) sind Räumlichkeiten vorhanden, die eine Differenzierung in dem Sinne erlauben, dass unterschiedliche Gruppen an unterschiedlichen Orten in verschiedenen Settings arbeiten können. Dies ist innerhalb des Klassenzim- 
mers der Fall, welches eher ein Ensemble von Räumen und funktional unterschiedlich eingerichteten Ecken darstellt. Auch innerhalb des Schulhauses ist die Varianz der Räumlichkeiten größer, als dies beim Schulhaus Breitenrain der Fall ist. Und vom räumlichen Potenzial hätte die Anlage so gewählt werden können, dass auch große Außenräume vorhanden wären, die individuelles Lernen einer heterogenen Kinderschar mit unterschiedlichen Interessen ermöglichen würden.

Die Rekonstruktion lässt jedoch keine Hinweise darauf erkennen, dass eine große Varianz von Lehr-Lern-Gelegenheiten vorhanden ist. Die Erzeugnisse der Schüler_innen - zum Beispiel die Dekorationen und die Bilder mit dem Schneemann -, aber auch die farbig markierten Arbeitsblätter weisen auf einen Unterricht hin, in dem das Prinzip der Serialität in der Vermittlung eines normierten, kodizifierten Wissens in normierten Wissensformen dominant ist. Es gibt keine Anzeichen dafür, dass Räume, Materialien und Artefakte vorhanden sind, die einer Heterogenität der Schüler_innen mit unterschiedlichen Potenzialen und mit unterschiedlichen Möglichkeiten, Interessen, Vorlieben sowie Bildungszielen entspräche. Es finden sich keine Anzeichen, dass autonome Aneignung erwünscht, geschweige denn gefördert würde, dass Schüler_innen Raum für eigene Ideen oder Interessen finden, wo sie diese umsetzen könnten. Im Gegenteil ist ein wesentliches rekonstruiertes Merkmal des Schulhauses, dass es gerade keine Räume für autonomes Lernen, für autonome Sozialisation und auch für einen zweckfreien Aufenthalt in den Peer-Kontakten zur Verfügung stellt. Sie werden durch manifeste architektonische Artefakte und deren latente Sinnstrukturen unterbunden.

Die äußere Gestalt und die innere Raumkonfiguration des Schulhauses sind insofern kongruent, als die Lernprozesse als individualisierte Aneignung über den Stimulus oder die Handlungsanweisungen von Artefakten initiiert werden. Die rekonstruierte Eigenlogik des Gebäudes ist auf eine individualisierte Aneignung von Routinen gerichtet: vom individuellen Üben von Wurftechniken bis zum individuellen Lösen von Arbeitsblättern. Dem ist einerseits die Möglichkeit zur direkten pädagogischen Interaktion nachgelagert. Andererseits sind die Anregungen, die das Gebäude und die Einrichtung bieten, eher minimal.

Jenseits der Frage, welche Anregungen Schulhäuser aus und in ihrer Architektur bieten können, ist festzuhalten, dass sowohl dem Einfluss der Architektur als auch jenem von Unterrichtsformen beziehungsweise -konzepten Grenzen gesetzt sind. Im Prinzip kann überall und in jedem Setting guter beziehungsweise 
schlechter Unterricht realisiert werden. Dem Schulhaus Hinter Gärten kann zumindest ansatzweise zugutegehalten werden, dass es einen relativ großen Möglichkeitsraum an Unterrichtssettings eröffnet. Dem widerspricht allerdings sein Werkcharakter, der einer Aneignung prinzipiell entgegensteht.

Mit der Schulanlage Hinter Gärten wurde rekonstruiert, dass ikonische Embleme der Schule durch das Gebäude und seine Farbigkeit verwischt werden. Auch als Gebäudetypus, verschwindet" die Schule als Schule insofern, als sie nicht mehr unmittelbar als solche oder durch darauf verweisende Embleme erkennbar ist.

Die Schule Hinter Gärten manifestiert sich als Schule in Form und Suggestion eines postfordistischen Industriebaus, der eine enge posttayloristische Verzahnung von geistiger Tätigkeit mit weitestgehend automatisierter Produktion verheißt. In diesem Sinne - und auch in dem Übertünchen der inneren Schulförmigkeit - repräsentiert sie eine Schule, die sich explizit von der Schulförmigkeit eines normierten Gebäudetypus distanziert, obwohl sie zu weiten Teilen diese Schulförmigkeit weiterhin herstellt. Es ist eine gewisse Analogie in der Figur zu sehen, wie sie Adorno (1969) in den „Tabus über den Lehrberuf“ ausführt. Dem Gebäude scheint eine gewisse Abneigung gegenüber seiner Funktion eingeschrieben, die es zu vertuschen gilt beziehungsweise die es als Kunstobjekt schöngeistig übertüncht.

Für einen nächsten kontrastierenden Fall werden die Schulhäuser von Schmitten im Kanton Freiburg analysiert. Sie erlauben erstens einen Überblick über die historische Genese und Brüche im Schulhausbau. Es sind fünf Schulhäuser aus unterschiedlichen bauhistorischen- und auch bildungspolitischen Etappen vorhanden. Zum Zweiten ist für die Schule in Schmitten bezeichnend, dass sie ihre Schulanlage mit einem parkartigen Bereich geöffnet hat, um auch Nicht-Schüler_innen anzusprechen.

Open Access Dieses Kapitel wird unter der Creative Commons Namensnennung 4.0 International Lizenz (http://creativecommons.org/licenses/by/4.0/deed.de) veröffentlicht, welche die Nutzung, Vervielfältigung, Bearbeitung, Verbreitung und Wiedergabe in jeglichem Medium und Format erlaubt, sofern Sie den/die ursprünglichen Autor(en) und die Quelle ordnungsgemäß nennen, einen Link zur Creative Commons Lizenz beifügen und angeben, ob Änderungen vorgenommen wurden.

Die in diesem Kapitel enthaltenen Bilder und sonstiges Drittmaterial unterliegen ebenfalls der genannten Creative Commons Lizenz, sofern sich aus der Abbildungslegende nichts anderes ergibt. Sofern das betreffende Material nicht unter der genannten Creative Commons Lizenz steht und die betreffende Handlung nicht nach gesetzlichen Vorschriften erlaubt ist, ist für die oben aufgeführten Weiterverwendungen des Materials die Einwilligung des jeweiligen Rechteinhabers einzuholen.

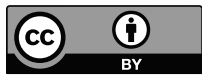

